\title{
Preventive, Behavioral, Productive, and Tissue Modification using Green Synthesized Selenium Nanoparticles in the Drinking Water of Two Broiler Breeds under Microbial Stress
}

\section{-Author(s)}

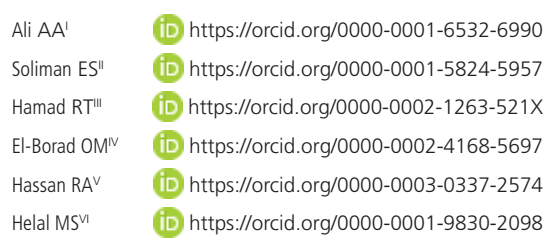

Animal Behavior and Management Division, Department of Animal Hygiene, Zoonosis, and Animal Behavior, Faculty of Veterinary Medicine, Suez Canal University, Ismailia, Egypt, 41522.

Animal, Poultry and Environmental Hygiene Division, Department of Animal Hygiene, Zoonosis, and Animal Behavior, Faculty of Veterinary Medicine, Suez Canal University, Ismailia, Egypt, 41522

III Department of Pathology, Faculty of Veterinary Medicine, Menoufia University, AL Minufya, Egypt, 32511.

Institute for Nanoscience and Nanotechnology, Kafrelsheikh University, Kafrelsheikh, Egypt, 33511.

Animal Production Division, Department of Animal Wealth Development, Faculty of Veterinary Medicine, Suez Canal University, Ismailia, Egypt, 41522.

Reference Laboratory for Veterinary Quality Control on Poultry Production (RLQP), Ismailia, Egypt, 41513 .

\section{-Mail Address}

Corresponding author e-mail address Essam S. Soliman

Department of Animal Hygiene, Zoonosis \& Animal Behavior, Faculty of Veterinary Medicine, Suez Canal University, Ismailia, Egypt, 41522.

Phone: 201006597914

Email: soliman.essam@vet.suez.edu.eg

\section{- Keywords}

Behavior, Broiler, Housing, Immunity, Nanoselenium.

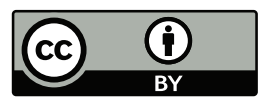

Submitted: 27/June/2019

Approved: 18/October/2019

\section{ABSTRACT}

A study was conducted to investigate the influence of selenium nanoparticles (SeNPS) and inorganic selenium supplementation in the drinking water on behavior, performance, and immunity of Arbor Acres $^{\circledR}$ and Ross $^{\circledR 3} 308$ broilers exposed to E. coli O157:H7 $1.6 \times 10^{8}$ challenge at the $10^{\text {th }}$ day of age.

180 one-day-old female broilers were divided into six groups, each with 30 chicks. G1 and G4 were supplied with $1 \mathrm{~mL}$ SeNPS $100 \mathrm{mg} \cdot \mathrm{L}^{-1} / \mathrm{L}$, $\mathrm{G} 2$ and $\mathrm{G} 5$ were supplied with $1 \mathrm{~mL}$ inorganic selenium $100 \mathrm{mg} \cdot \mathrm{L}^{-1} / \mathrm{L}$, and $\mathrm{G} 3$ and $\mathrm{G} 6$ were supplied with non-supplemented water. Where, G1, G2, and G3 were Arbor Acres $^{\circledR}$, while G4, G5, and G6 were Ross ${ }^{\circledR 3} 38$ broilers. A total of 1280 samples (160 sera, 160 intestinal swabs and 960 organ samples including liver, spleen, bursa, heart, breast muscles, and thymus) were collected in a study period of 38 days.

A highly significant increase $(p<0.01)$ of weight gain, feed conversion, performance index, total protein, albumin, alanine aminotransferase, aspartate aminotransferase, immunoglobulin $\mathrm{G}$ and $\mathrm{M}$, total antioxidant capacity, malondialdehyde, and superoxide dismutase was recorded in G4 Ross ${ }^{\circledR 3} 08$ compared to other supplemented and control groups. G1 Arbor Acres $^{\circledR}$ and G4 Ross $^{\circledR 3} 308$ broilers, also revealed a highly significant decline $(p<0.01)$ in total bacterial and enterobacteriaceae counts of intestine and breast muscles compared to G2 Arbor Acres $^{\circledR}$ and G5 Ross ${ }^{\circledR} 308$ broilers and to controls. Photomicrographs revealed a higher degree of cellular and tissue protection in G4 and G5 Ross ${ }^{\circledR} 308$ compared to $\mathrm{G} 1$ and G2 Arbor Acres ${ }^{\circledR}$ broilers. An improvement from SeNPS supplementation was detected on behavior, performance, bacterial load, immunological, antioxidant profiles, and tissue architecture in broilers breeds with special reference to Ross ${ }^{\circledR 3} 38$ compared to Arbor Acres ${ }^{\circledR}$ broilers.

\section{INTRODUCTION}

The poultry industry worldwide has witnessed large scale intensification proportional to the growing population. Intensification in poultry industry demands proactive preventive measures and enhancing supplemental to maintain high productivity, minimize disease risk, and lessen the influence of environmental stressors. Rearing broilers under adverse conditions produce oxidative stress, including extreme freeradical formation and dispersion, cell destruction, and production losses (Schwean-Lardner et al., 2012). It was established that using complexes containing vitamins, minerals, and selenium improved productivity, increased weight gain by $8.46 \%$, and improved feed conversion by 3.1\% (Trukhachev et al., 2016).

Selenium is a nutritionally essential for chickens, to maintain an optimum health, therefore $0.15 \mathrm{mg} / \mathrm{kg}$ diet is suggested for broilers during the growth period. Selenium is an important structure of 
Ali AA, Soliman ES, Hamad RT, El-Borad OM, Hassan RA, Helal MS
Preventive, Behavioral, Productive, and Tissue Modification using Green Synthesized Selenium Nanoparticles in the Drinking Water of Two Broiler Breeds under Microbial Stress

\section{Experimental birds' microclimate}

A total of 180 one-day-old females Arbor Acres ${ }^{\circledR}$ and $\operatorname{Ross}^{\circledR 3} 308$ broilers (ninety of each breed) were purchased from Ismailia-Egypt Poultry Company and housed in a battery system (galvanized iron cages). Birds of each breed were divided into three groups; each group consisted of 30 chicks (five replicates of six birds).

The birds were immunized be mean of mass vaccination in drinking water with Infectious Bronchitis live attenuated virus vaccine (PESTIKAL B1 SPF 1000 dose/ 100 Liter) on the $7^{\text {th }}$ day of age; Infectious Bursal disease live attenuated virus vaccine (SER-VAC D78 Strain 1000 dose/ 100 Liter) on the $14^{\text {th }}$ and $21^{\text {st }}$ days of age, and Newcastle disease live lentogenic virus vaccine (PESTIKAL 1000 dose/100 Liter) on the $16^{\text {th }}$ and $26^{\text {th }}$ days. The experiment lasted for 38 days.

At day-old the rearing temperature was $34{ }^{\circ} \mathrm{C}$ and gradually declined by $3.5^{\circ} \mathrm{C}$ weekly until achieving 25 ${ }^{\circ} \mathrm{C}$ by the end of the 3rd week. The birds were given ad libitum access to water and a standard corn-soybean basal diet (NRC 1994) based on $23 \%$ crude protein over the first fourteen days, and $21.5 \%$ crude protein for the remaining 24 days in the fattening cycle. The room was ventilated naturally using $V$ shape windows to stimulate a stack effect. Artificial continuous lightening program for $18 \mathrm{~h}$ light and $6 \mathrm{~h}$ darkness using Blue LED lights was provided as recommended by Soliman \& Hassan (2019). Mortalities, microclimatic temperature and relative humidity were monitored and recorded daily during the experiment.

\section{Synthesis of Selenium nanoparticles}

Selenium powder $\left(\right.$ Merk $\left.^{\circledR}\right)$ was used as a precursor for the SeNPS and polyvinyl alcohol (PVA) served as a capping agent for the NPS to prevent aggregation and any change in size and shape. Glucose that was commercially produced acts as a reducing agent of selenium; it is assumed that glucose makes reduction and oxidized itself into gluconic acid, this acid may also surround the NPS and act as a stabilizing agent. Firstly aqueous sodium seleno-sulphate solution was prepared by refluxing and heating at $70{ }^{\circ} \mathrm{C}$ for an aqueous solution of $\mathrm{Na}_{2} \mathrm{SO}_{3}\left(\mathrm{Merk}^{\circledR}\right)$ and selenium powder (selenite) for $6 \mathrm{~h}$ as reported in Gorer \& Hodes (1994). The solution was filtered to remove any unreacted materials, then glucose powder $(6 \%)$ and $1 \%$ PVA were added to the solution, refluxing persisted for another $6 \mathrm{~h}$. The color of the solution changed through refluxing from colorless to pale yellow. The colored solution remained stable for months. The synthesized SeNPS were characterized by UV-visible 
Ali AA, Soliman ES, Hamad RT, El-Borad OM, Hassan RA, Helal MS
Preventive, Behavioral, Productive, and Tissue Modification using Green Synthesized Selenium Nanoparticles in the Drinking Water of Two Broiler Breeds under Microbial Stress optical absorption spectroscopy (Shimaduz RF5301PC double beam spectrophotometer), and absorption spectra of the resulting SeNPS were scanned (200-800 $\mathrm{cm}-1$ ) using $1 \mathrm{~cm}$ matched quartz cells. The morphology of the NPS was observed using Transmission Electron Microscopy (HR-TEM) JOEL JEM-2010 operating at an accelerating voltage of $200 \mathrm{kV}$ and equipped with a Gatan digital camera Erlangshen ES500. TEM sample was prepared by dropping a sample suspension as it is after preparation on a coppergrid coated with a carbon film. Preparing SeNPS via this method characterized by obtaining Nanocrystalline SeNPS without post annealing treatment.

\section{Selenium and SeNPS solution supplement}

One group ( $\mathrm{N}=30)$ from each breed was assigned the following treatments: $\mathrm{G} 1$ and $\mathrm{G} 4$ were supplied with 1 $\mathrm{mL}$ SeNPS (green synthesized SeNPS at concentration of $100 \mathrm{mg} / \mathrm{L}$ ) per each liter of drinking water, G2 and G5 were supplied with $1 \mathrm{~mL}$ commercial inorganic selenium (Selenite - SEDICO ${ }^{\circledR}$ at concentration of $100 \mathrm{mg} / \mathrm{L}$ ) per each liter of drinking water, finally, G3 and G6 were supplied with non-supplemented water. Where, G1, G2, and G3 were Arbor Acres ${ }^{\circledR}$, G4, G5, and $G 6$ were Ross ${ }^{\circledR 3} 08$ broilers. The final concentration of both SeNPS and inorganic selenium in the supplemented water was about $0.1 \mathrm{mg}$ per each liter of drinking water on a daily basis of the entire fattening cycle (38 days).

\section{E. coli 0157:H7 challenge}

E. coli 0157: H73.6 × $10^{5} \mathrm{CFU}$ culture was purchased from the Animal Health Research Institute - Dokki, the culture was propagated using Mac-Conkey broth at $44{ }^{\circ} \mathrm{C} / 24 \mathrm{~h}$. Ten microliters from the positive tubes (yellow color development and gas accumulation in inverted Durham's tube) were dropped aseptically onto Eosine Methylene Blue (EMB) agar, and incubated at $37{ }^{\circ} \mathrm{C} / 24 \mathrm{~h}$ (Herigstad et al., 2001). Metallic green colonies were counted and picked up. Four out of the six groups (G1, G2, G4 and G5) were challenged with E. Coli 0157:H7 $1.6 \times 10^{8}$ on the $10^{\text {th }}$ day of age in drinking water (Kocijancic et al., 2016).

\section{Performance indices}

Average live body weight (LBW) was estimated by weighing 28 birds (the number was calculated using Solvin's formula for random sampling) from each group on a weekly basis. Weekly feed intake (FI) was calculated based on total amount consumed in relation to the bird's absolute number in each group. Body Weight Gain (WG), Feed Conversion Ratio (FCR), and Performance Index (PI) were calculated according to Soliman \& Hassan (2017).

\section{Behavior recording and observation methods}

Twelve broiler chicks from each group were randomly selected for recording the behavioral parameters. Broiler's behavior was recorded for a total of $3 \mathrm{~h}$ a day, $1 \mathrm{~h}$ starting at 9:00 am, 2:00 pm, and 9:00 pm in a daily pattern for three weeks $\left(1\right.$ st, $3^{\text {rd }}$, and the $5^{\text {th }}$ ) using a video camera (Panasonic WV Ns202ae) connected to computer through DVR card, and they were suspended at a height of $1.5 \mathrm{~m}$ above the floor of each pen (Li et al., 2015).

The recorded videos were replayed in the laboratory, and the performed behaviors, duration, and frequency were recorded every five $\mathrm{min} / \mathrm{h}$ (12 observations per h) using focal sampling method. They were classified into: feeding, drinking, walking, standing, resting, preening, stretching, pecking, and flapping (Villagra et al., 2014).

\section{Sampling}

Sampling was carried out via two slaughters (at the $19^{\text {th }}$ and the $38^{\text {th }}$ day of age). A total of 1280 samples were collected during the study period, including 160 sera, 160 intestinal swabs and 960 organ samples of liver, spleen, bursa, heart, breast muscles, and thymus. Blood samples were collected during slaughters, centrifuged at $4000 \mathrm{rpm}$ for 15 minutes. Nonhemolyzed clear sera were obtained, stored at $-20^{\circ} \mathrm{C}$ for the biochemical and antioxidant assay (Soliman et al., 2017). Liver, spleen, bursa, heart, and thymus were kept on formalin 10\% for histopathological examination. Intestinal swabs, and $3 \mathrm{~g}$ of breast muscles were collected in $9 \mathrm{~mL}$ buffered peptone water for bacteriological assessment.

\section{Biochemical profile and antioxidant markers}

Sera samples were examined for total protein (TP), albumin (ALB), Alanine aminotransferase (ALT), Aspartate aminotransferase (AST), urea (UREA), and creatinine (CREAT), as well as for antioxidant markers including: Total Antioxidant Capacity (TAC), Malondialdehyde (MDA), and Superoxide Dismutase (SOD) calorimetrically using UV-1100 spectrophotometer.

Serum immunoglobulin Ig $\mathrm{G}$ and IgM concentrations were measured by using immunoturbidimetric assay using Sysmex CS-5100 System. 
Ali AA, Soliman ES, Hamad RT, El-Borad OM, Hassan RA, Helal MS
Preventive, Behavioral, Productive, and Tissue Modification using Green Synthesized Selenium Nanoparticles in the Drinking Water of Two Broiler Breeds under Microbial Stress

\section{Bacteriological examination}

Intestinal swabs, as well as breast muscles samples, were prepared according to APHA (2012). Tenfold serial dilution sup to $10^{-6}$ were prepared. Total Bacterial Count (TBC) and Total Enterobacteriaceae Count (TEC) were applied on standard plate count (SPC) and Eosine Methylene Blue Agar (EMB), respectively at $37{ }^{\circ} \mathrm{C}$ for $24-48 \mathrm{~h}$ by using the drop plate technique as recommended by Soliman et al. (2016) and Kim \& Lee (2016). Plates were counted using Dark-field colony counter (Murray et al., 2015).

\section{Histopathological examination}

Representative tissue samples from liver, heart, lymphoid organs (spleen, bursa of Fabricious, and thymus) were fixed in 10\% buffered formalin saline solution until further processing. Specimens were cut into 5-mm thick sections, put into tissue cassettes, dehydrated by transferring through a series of alcohols increasing concentrations, cleared in two changes of xylene, infiltrated with different grades of melted paraffin in the oven, and finally the sections were cut at $5 \mu \mathrm{m}$ thickness using rotator microtome. After cutting, sections were floated on warm water bath at $38^{\circ} \mathrm{C}$ for stretching, mounted on clean slides using egg albumin, and dried on a slide warmer at $38^{\circ} \mathrm{C}$ (Luna, 1968 and Darboux, 1994). The sections were stained using Hematoxylin and Eosin ( $\mathrm{H} \& \mathrm{E})$. The histological structures of the examined organs were observed using light microscope under (x10) and (x20) magnification.

\section{Statistical analysis}

Statistical analysis was carried out using the statistical package for social sciences (SPSS version 20.0) software package (Argyrous, 2005). E. coli 0157:H7 and total bacterial counts were expressed as logarithms using Microsoft Excel. The obtained data were analyzed statistically using two-tailed Analysis of Variance (ANOVA). In the two-tailed ANOVA, factors including breeds, groups, age of broiler, and slaughter times were used as between-subjects' effects along with their interactions. Mixed model ANOVA was used as the principal statistical mean for the current data. The principle and interaction effects were tested for their statistical significance at 0.05 and 0.01 significance levels. The statistical model was emphasized as following:

$$
Y_{i j k}=\mu+\alpha_{1}+\beta_{j}+(\alpha \beta)_{i j}+e_{i j k}
$$

Where, $Y_{i j k}$ was the measurement of any dependent variable; $\mu$ was the overall mean; $\alpha_{1}$ was the fixed effect of slaughter time; $B_{j}$ was the fixed effect of the age of the birds; $(\alpha \beta)_{i j}$ was the interaction effect of slaughter time by age of the birds; $\mathrm{e}_{\mathrm{ijk}}$ was the random error. The error term, $e_{i j k}$ was approximately NID (0, $\sigma^{2}{ }_{\mathrm{e}}$ ), normally independently distributed with mean of 0 and variance of, $\sigma_{e}^{2}$.

\section{RESULTS}

\section{UV-Vis spectrophotometry of SeNPS}

The absorption spectrum of yellow colored SeNPS (Figure 1) showed appearance of the transition point at around $367 \mathrm{~nm}$, without a clear maximum reflecting the formation of SeNPS. The stabilization of the yellow color during the entire reaction time supported the stabilizing influence of PVA. Morphology and structure of the synthesized SeNPS was observed using TEM imaging, and the image (Figure 2) revealed a spherical shape for individual nanoparticles, with size at $25 \mathrm{~nm}$.

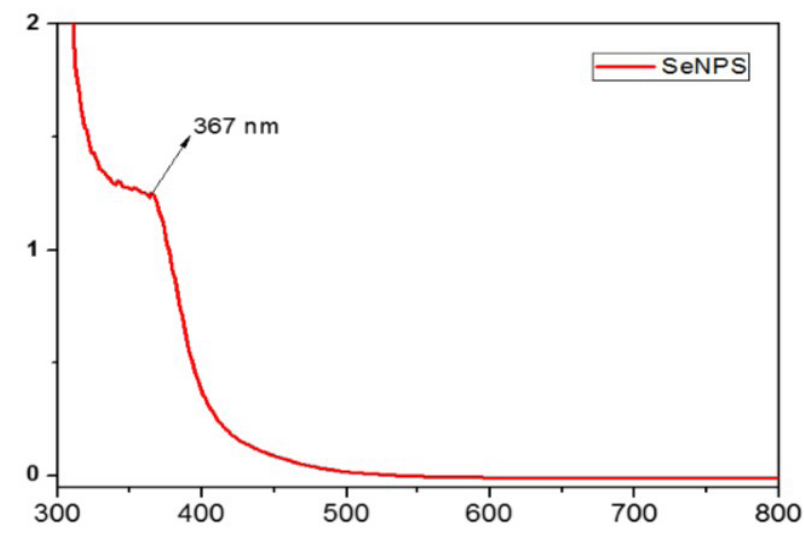

Figure 1 - The absorption spectrum of prepared SeNPS.

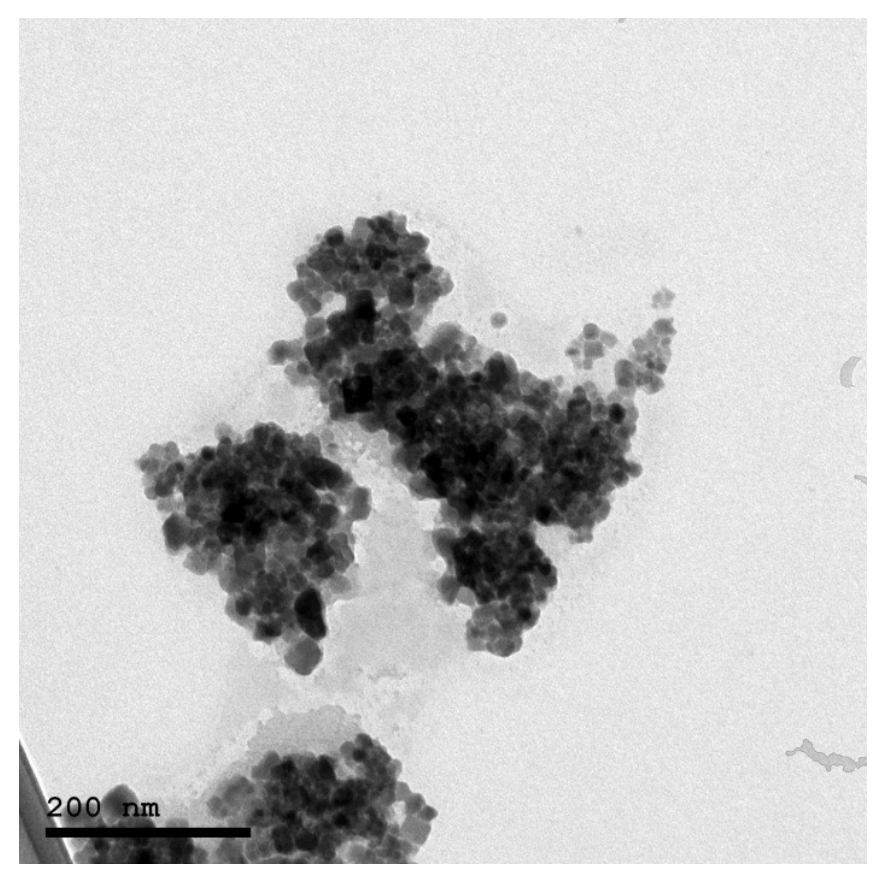

Figure 2 - The TEM image for the prepared SeNPS. 
Ali AA, Soliman ES, Hamad RT, El-Borad OM, Hassan RA, Helal MS
Preventive, Behavioral, Productive, and Tissue Modification using Green Synthesized Selenium Nanoparticles in the Drinking Water of Two Broiler Breeds under Microbial Stress

\section{Behavioral observations}

Broiler's behavior (Table 1) exhibited a highly significant increase $(p<0.01)$ in the frequencies of feeding, drinking, walking, resting, and in the duration of drinking and walking in G1 and G4 supplemented with SeNPS compared to $G 2$ and $G 5$ supplemented with commercial inorganic selenium and to the controls. A highly significant decrease $(p<0.01)$ was recorded in the duration of feeding and standing among all treated groups in both broilers' breeds compared to the control. A highly significant increase $(p<0.01)$ was declared in walking and drinking duration of $\mathrm{G} 1$ and $\mathrm{G} 4$ compared to all other supplemented and control groups.

SeNPS (Table 1) were able to produce a highly significant decrease $(p<0.01)$ in standing duration of G1 and G4 when compared to each other, a nonsignificant decrease in standing duration of $\mathrm{G} 2$ and $\mathrm{G} 5$, and a non-significant decrease in standing frequency among all groups of both breeds.

Resting frequency showed (Table 1) a highly significant increase $(p<0.01)$ in $\mathrm{G} 1$ broilers compared to $G 2, G 5$, and $G 4$ with no significant difference between theses latter three groups. The selenium effect was inconsistent having no effect on resting frequency in either breed.

SeNPS was able to significantly decrease $(p<0.01)$ the frequencies of preening, stretching, and pecking in G1, G4, G2, and G5 compared to their controls (Table
1). On the other hand, it was able to increase the frequency of flapping in the same groups compared to their controls.

\section{Biochemical profile}

The biochemical profile (Table 2 ) revealed a highly significant decrease $(p<0.01)$ in TP, ALB, ALT, and AST among treated groups of both experimented broilers' breeds compared to their control. TP revealed (Table 2 ) a highly significant elevation $(p<0.01)$ in $\mathrm{G} 4$ Ross ${ }^{\circledR 3} 08$ compared to G1Arbor Acres ${ }^{\circledR}$ and to the other treated groups. ALB showed a highly significant increase $(p<0.01)$ in $G 5$ Ross $^{\circledR} 308$ broilers treated with inorganic selenium compared to G2 Arbor Acres ${ }^{\circledR}$ broilers treated with the same dose and concentration of inorganic selenium, and to $\mathrm{G} 1$ and $\mathrm{G} 4$ treated with SeNPS.

On a time scale, TP showed a highly significant decrease $(p<0.01)$ on the 38 day old broilers compared to the 19 day old broilers in G1, G2, G3, and G4, and a highly significant increase on the 38 day old broilers compared to the 19 day old broilers in G5 and G6 (Table $2)$. ALB revealed a highly significant decrease $(p<0.01)$ on the 38 day old broilers compared to the 19 day old in $\mathrm{G} 1, \mathrm{G} 2, \mathrm{G} 4$, and $\mathrm{G} 5$, no significant differences between the two slaughter times in $\mathrm{G} 3$ Arbor Acres ${ }^{\circledR}$ control, and a highly significant increase $(p<0.01)$ on the 38 day old broilers compared to the 19 day old in G6 Ross ${ }^{\circledR 3} 308$ control (Table 2).

Table 1 - Behavioral patterns of different broiler breeds supplemented with selenium and green synthetized selenium nanoparticles.

\begin{tabular}{|c|c|c|c|c|c|c|c|c|c|}
\hline \multirow[t]{2}{*}{ Groups } & \multicolumn{9}{|c|}{ Behaviors } \\
\hline & Feeding & Drinking & Walking & Standing & Resting & Preening & Stretching & Pecking & Flapping \\
\hline \multicolumn{10}{|c|}{ Duration } \\
\hline G1 & $137.5^{\mathrm{e}} \pm 25.3$ & $67.5^{b} \pm 16.2$ & $80.8^{a} \pm 20.13$ & $9.1^{d} \pm 3.58$ & $614.1^{\mathrm{a}} \pm 29.9$ & |--------- & 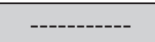 & ---------" & ----------- \\
\hline G2 & $148.3^{d} \pm 37.9$ & $66.6^{b} \pm 5.12$ & $81.6^{a} \pm 23.86$ & $27.5^{\mathrm{ab}} \pm 7.60$ & $575.8^{a} \pm 48.6$ & ---------- & ---------- & ----------- & ---------- \\
\hline G3 & $163.3^{c} \pm 46.9$ & $58.7^{c} \pm 17.6$ & $40.8 \pm \pm 9.25$ & $33.3^{a} \pm 13.10$ & $593.3^{a} \pm 56.2$ & ----------- & ----------- & ----------- & ----------- \\
\hline G4 & $205.0^{b} \pm 30.8$ & $95.0^{a} \pm 19.6$ & $40.0^{c} \pm 6.51$ & $7.5^{\mathrm{d}} \pm 2.18$ & $535.0^{\mathrm{a}} \pm 35.0$ & ----------- & ----------- & ---------- & ----------- \\
\hline G5 & $214.1^{b} \pm 46.8$ & $93.3^{\mathrm{a}} \pm 30.8$ & $50.0^{b} \pm 11.28$ & $27.5^{\mathrm{ab}} \pm 8.36$ & $551.6^{a} \pm 62.1$ & ----------- & ----------- & ----------- & ----------- \\
\hline G6 & $230.0^{a} \pm 51.5$ & $61.6^{b c} \pm 14.7$ & $20.0^{d} \pm 5.37$ & $30.0^{a b} \pm 10.3$ & $552.5^{a} \pm 55.9$ & ----------- & ----------- & ----------- & ----------- \\
\hline$p$ value & 0.002 & 0.000 & 0.005 & 0.001 & 0.330 & --------- & ---------- & -------- & --------- \\
\hline \multicolumn{10}{|c|}{ Frequency } \\
\hline G1 & $1.83^{b} \pm 0.34$ & $2.17^{\mathrm{a}} \pm 0.73$ & $3.83^{\mathrm{a}} \pm 0.41$ & $0.83 \pm 0.32$ & $4.17^{a} \pm 0.37$ & $0.58^{e} \pm 0.15$ & $0.75^{b} \pm 0.22$ & $0.67 \pm 0.45$ & $0.50^{b} \pm 0.23$ \\
\hline G2 & $1.75^{b} \pm 0.33$ & $1.92^{b} \pm 0.19$ & $3.92^{\mathrm{a}} \pm 0.60$ & $1.50 \pm 0.31$ & $3.17^{b} \pm 0.24$ & $1.00^{c} \pm 0.43$ & $1.08^{\mathrm{a}} \pm 0.31$ & $0.42^{d} \pm 0.23$ & $0.97^{a} \pm 0.58$ \\
\hline G3 & $1.42^{c} \pm 0.34$ & $1.42^{\mathrm{d}} \pm 0.29$ & $2.50^{b} \pm 0.42$ & $1.00 \pm 0.37$ & $3.25^{b} \pm 0.25$ & $1.67^{a} \pm 0.45$ & $1.08^{\mathrm{a}} \pm 0.29$ & $1.50^{\mathrm{a}} \pm 0.93$ & $0.17^{d} \pm 0.11$ \\
\hline G4 & $2.25^{\mathrm{a}} \pm 0.22$ & $1.83^{b} \pm 0.24$ & $2.00^{\wedge} \pm 0.33$ & $0.75 \pm 0.18$ & $3.42^{b} \pm 0.23$ & $0.42^{\mathrm{e}} \pm 0.29$ & $0.17^{d} \pm 0.11$ & $0.75^{c} \pm 0.51$ & $0.25^{c} \pm 0.13$ \\
\hline G5 & $2.08^{a} \pm 0.40$ & $1.50^{\complement} \pm 0.38$ & $1.83^{c} \pm 0.30$ & $0.58 \pm 0.15$ & $2.67^{c} \pm 0.19$ & $0.75^{d} \pm 0.00$ & $0.33^{c} \pm 0.19$ & $0.00^{e} \pm 0.00$ & $0.18^{d} \pm 0.02$ \\
\hline G6 & $1.75^{b} \pm 0.33$ & $1.088^{e} \pm 0.19$ & $1.17^{d} \pm 0.30$ & $1.00 \pm 0.25$ & $2.92^{c} \pm 0.29$ & $1.28^{b} \pm 0.00$ & $0.33^{c} \pm 0.14$ & $0.92^{b} \pm 0.64$ & $0.17^{d} \pm 0.11$ \\
\hline$p$ value & 0.001 & 0.000 & 0.000 & 0.086 & 0.005 & 0.023 & 0.000 & 0.006 & 0.023 \\
\hline
\end{tabular}

Means carrying different superscripts in the same column are significantly different at $(p \leq 0.05)$ or highly significantly different at $(p<0.01)$. Means carrying the same superscripts in the same column are non-significantly different at $(p<0.05)$.

$\mathrm{G} 1=$ Arbor Acres ${ }^{\circledR}$ with $1 \mathrm{~mL}$ nano-selenium/1L drinking water, G2=Arbor Acres ${ }^{\circledR}$ with $1 \mathrm{~mL}$ selenium/1L drinking water), G3=Arbor Acres ${ }^{\circledR}$ control, G4=Ross $^{\circledR} 308$ with $1 \mathrm{~mL}$ nano-selenium $/ 1 \mathrm{~L}$ drinking water, $\mathrm{G} 5=$ Ross $^{\circledR} 308$ with $1 \mathrm{~mL}$ selenium/1 1 drinking water, and $\mathrm{G} 6=$ Ross $^{\circledR} 308$ control. 
A highly significant decline $(p<0.01)$ was detected (Table 2) in ALT of G1 and G2 Arbor Acres ${ }^{\circledR}$ broilers compared to their control with no significant differences between these two groups, and a highly significant decline $(p<0.01)$ of ALT in G4 and G5 Ross ${ }^{\circledR 3} 08$ broilers compared to their control, with a highly significant increase $(p<0.01)$ in $\mathrm{G} 4$ than in $\mathrm{G} 5$ broilers. A highly significant decrease $(p<0.01)$ was revealed in AST of G1 treated with SeNPS compared to $G 2$ treated with inorganic selenium and $\mathrm{G} 3$ control Arbor Acres ${ }^{\circledR}$ broilers, and in AST of G4 treated with SeNPS compared to G5 treated with inorganic selenium and G6 control Ross ${ }^{\circledR 3} 308$ broilers.

ALT showed a highly significant decrease $(p<0.01)$ on the 38 day old broilers compared to the 19 day old in $\mathrm{G} 1, \mathrm{G} 3$, and $\mathrm{G} 4$, no significant differences between the two slaughter times in $\mathrm{G} 5$, and a highly significant increase $(p<0.01)$ on the 38 day old broilers compared to the 19 day old in $G 2$ and $G 6$ (Table 2). AST showed a highly significant increase $(p<0.01)$ on the 38 day old broilers compared to the 19 day old in all treated and control groups (Table 2).

Urea (Table 2) showed a highly significant increase $(p<0.01)$ in $\mathrm{G} 1$ Arbor Acres $^{\circledR}$ and G5 Ross $^{\circledR 308}$, nonsignificant increase in $\mathrm{G} 2$ Arbor Acres $^{\circledR}$, and a highly significant decrease $(p<0.01)$ in $\mathrm{G} 4$ Ross $^{\circledR} 308$ compared to the controls. A highly significant increase $(p<0.01)$ of CREAT levels was noticed in G1, G2, G4, and G5 compared to their controls.

Urea revealed a highly significant decrease $(p<0.01)$ on the 38 day old broilers compared to the 19 day old in $\mathrm{G} 3, \mathrm{G} 5$, and $\mathrm{G} 6$, no significant differences between the two slaughter times in $\mathrm{G} 1$, and a highly significant increase $(p<0.01)$ on the 38 day old broilers compared to the 19 day old in $\mathrm{G} 2$ and $\mathrm{G} 4$ (Table 2). CREAT showed a highly significant decrease $(p<0.01)$ on the 38 day old broilers compared to the 19 day old in $\mathrm{G} 1$, no significant differences between the two slaughter times in $G 6$, and a highly significant increase $(p<0.01)$ on the 38 day old broilers compared to the 19 day old in G2, G3, G4 and G5 (Table 2).

Table 2 - Biochemical profile (Mean \pm SE) of different broiler breeds supplemented with selenium and green synthetized selenium nanoparticles.

\begin{tabular}{|c|c|c|c|c|c|c|c|}
\hline 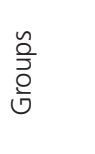 & 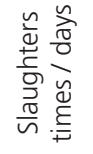 & $\begin{array}{l}\text { TP } \\
\text { g/dl }\end{array}$ & $\begin{array}{l}\text { ALB } \\
\mathrm{g} / \mathrm{dl}\end{array}$ & $\begin{array}{l}\text { ALT } \\
I U / L\end{array}$ & $\begin{array}{l}\text { AST } \\
\text { IU/L }\end{array}$ & $\begin{array}{l}\text { UREA } \\
\mathrm{mg} / \mathrm{dl}\end{array}$ & $\begin{array}{l}\text { CREAT } \\
\mathrm{mg} / \mathrm{dl}\end{array}$ \\
\hline \multicolumn{2}{|l|}{ G1 } & $11.1^{c} \pm 0.26$ & $1.9^{c} \pm 0.13$ & $4.5^{b} \pm 0.20$ & $38.4^{b c} \pm 0.79$ & $46.8^{\mathrm{a}} \pm 1.34$ & $4.7^{\mathrm{a}} \pm 0.17$ \\
\hline \multicolumn{2}{|l|}{ G2 } & $10.1^{d} \pm 0.25$ & $1.8^{c} \pm 0.12$ & $4.5^{b} \pm 0.19$ & $46.3^{\mathrm{a}} \pm 0.74$ & $41.6^{b} \pm 1.27$ & $3.2^{b} \pm 0.16$ \\
\hline \multicolumn{2}{|l|}{ G3 } & $11.9^{b} \pm 0.26$ & $3.6^{a} \pm 0.13$ & $5.1^{\mathrm{a}} \pm 0.20$ & $39.3^{b} \pm 0.79$ & $40.3^{b} \pm 1.34$ & $0.8^{d} \pm 0.09$ \\
\hline \multicolumn{2}{|l|}{ G4 } & $11.7^{b} \pm 0.26$ & $1.5^{c} \pm 0.12$ & $2.8^{d} \pm 0.19$ & $33.3^{\mathrm{d}} \pm 0.77$ & $32.4^{d} \pm 1.31$ & $3.0^{b} \pm 0.17$ \\
\hline \multicolumn{2}{|l|}{ G5 } & $10.4^{\mathrm{d}} \pm 0.24$ & $2.7^{b} \pm 0.11$ & $2.3^{e} \pm 0.18$ & $36.3^{b c} \pm 0.72$ & $49.2^{\mathrm{a}} \pm 1.22$ & $2.2^{\complement} \pm 0.16$ \\
\hline \multicolumn{2}{|l|}{ G6 } & $12.4^{a} \pm 0.26$ & $3.4^{\mathrm{a}} \pm 0.12$ & $3.5^{c} \pm 0.19$ & $42.3^{b} \pm 0.77$ & $37.0^{c} \pm 1.31$ & $0.5^{d} \pm 0.02$ \\
\hline \multicolumn{2}{|l|}{$p$ value } & 0.000 & 0.000 & 0.002 & 0.005 & 0.004 & 0.005 \\
\hline \multicolumn{8}{|c|}{ Groups * Slaughter times } \\
\hline \multirow[t]{2}{*}{ G1 } & $19^{\text {th }}$ & $13.8^{a} \pm 0.46$ & $3.5^{\mathrm{a}} \pm 0.07$ & $5.6^{\mathrm{a}} \pm 0.73$ & $16.0^{\mathrm{b}} \pm 0.92$ & $45.4^{\mathrm{a}} \pm 1.03$ & $6.6^{\mathrm{a}} \pm 0.76$ \\
\hline & $38^{\text {th }}$ & $8.3^{b} \pm 0.49$ & $0.4^{b} \pm 0.04$ & $3.5^{b} \pm 0.18$ & $60.8^{a} \pm 0.50$ & $48.1^{a} \pm 0.46$ & $2.7^{b} \pm 0.14$ \\
\hline \multirow[t]{2}{*}{ G2 } & $19^{\text {th }}$ & $13.4^{\mathrm{a}} \pm 0.75$ & $2.9^{a} \pm 0.66$ & $4.3^{b} \pm 0.47$ & $23.2^{b} \pm 2.17$ & $14.7^{b} \pm 2.36$ & $1.6^{\mathrm{b}} \pm 0.16$ \\
\hline & $38^{\text {th }}$ & $6.9^{b} \pm 0.25$ & $0.7^{b} \pm 0.03$ & $4.7^{\mathrm{a}} \pm 0.19$ & $69.4^{a} \pm 0.74$ & $68.5^{a} \pm 2.18$ & $4.8^{a} \pm 0.21$ \\
\hline \multirow[t]{2}{*}{ G3 } & $19^{\text {th }}$ & $12.8^{a} \pm 0.67$ & $3.6^{a} \pm 0.08$ & $5.7^{\mathrm{a}} \pm 0.49$ & $9.8^{\mathrm{b}} \pm 1.10$ & $53.6^{\mathrm{a}} \pm 4.81$ & $0.5^{b} \pm 0.07$ \\
\hline & $38^{\text {th }}$ & $11.1^{b} \pm 0.06$ & $3.6^{a} \pm 0.02$ & $4.4^{b} \pm 0.05$ & $68.7^{\mathrm{a}} \pm 0.64$ & $27.1^{b} \pm 0.33$ & $1.1^{\mathrm{a}} \pm 0.05$ \\
\hline \multirow[t]{2}{*}{ G4 } & $19^{\text {th }}$ & $12.6^{a} \pm 0.42$ & $2.5^{\mathrm{a}} \pm 0.47$ & $4.7^{a} \pm 0.57$ & $11.0^{\mathrm{b}} \pm 2.08$ & $22.3^{b} \pm 2.59$ & $2.0^{\mathrm{b}} \pm 0.31$ \\
\hline & $38^{\text {th }}$ & $10.9^{b} \pm 0.08$ & $0.5^{b} \pm 0.01$ & $0.9^{b} \pm 0.04$ & $55.6^{a} \pm 1.15$ & $42.5^{\mathrm{a}} \pm 0.72$ & $4.1^{\mathrm{a}} \pm 0.15$ \\
\hline \multirow[t]{2}{*}{ G5 } & $19^{\text {th }}$ & $9.4^{b} \pm 0.69$ & $3.3^{\mathrm{a}} \pm 0.20$ & $2.5^{a} \pm 0.29$ & $12.5^{b} \pm 0.77$ & $51.1^{\mathrm{a}} \pm 3.31$ & $0.5^{b} \pm 0.07$ \\
\hline & $38^{\text {th }}$ & $11.4^{a} \pm 0.14$ & $2.2^{b} \pm 0.08$ & $2.1^{a} \pm 0.07$ & $60.1^{a} \pm 0.78$ & $47.2^{b} \pm 0.87$ & $3.9^{a} \pm 0.19$ \\
\hline \multirow[t]{2}{*}{ G6 } & $19^{\text {th }}$ & $10.8^{b} \pm 0.40$ & $1.9^{b} \pm 0.11$ & $2.1^{b} \pm 0.29$ & $12.6^{b} \pm 1.54$ & $45.2^{\mathrm{a}} \pm 1.03$ & $0.7^{a} \pm 0.08$ \\
\hline & $38^{\text {th }}$ & $14.0^{\mathrm{a}} \pm 0.12$ & $5.0^{\mathrm{a}} \pm 0.02$ & $4.9^{\mathrm{a}} \pm 0.08$ & $72.0^{\mathrm{a}} \pm 0.25$ & $28.9^{b} \pm 0.44$ & $0.3^{a} \pm 0.04$ \\
\hline \multicolumn{2}{|l|}{$p$ value } & 0.000 & 0.000 & 0.000 & 0.000 & 0.000 & 0.000 \\
\hline
\end{tabular}

Means carrying different superscripts in the same column are significantly different at $(p \leq 0.05)$ or highly significantly different at $(p<0.01)$. Means carrying the same superscripts in the same column are non-significantly different at $(p<0.05)$.

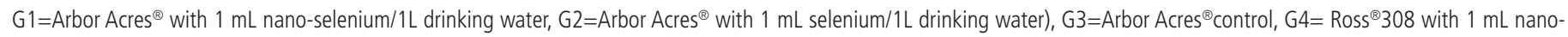
-selenium/1 L drinking water, $\mathrm{G} 5=$ Ross ${ }^{\circledR 3} 308$ with $1 \mathrm{~mL}$ selenium/1 L drinking water, and $\mathrm{G} 6=$ Ross ${ }^{\circledR} 308$ control.

$T P=$ Total Protein, $A L B=A l b u m i n, G L O B=G l o b u l i n, A L T=$ Alanine aminotransferase, $A S T=$ Aspartate aminotransferase, UREA=Urea, and $C R E A T=C r e a t i n i n e . ~ S E=S t a n d a r d$ error. 


\section{Immunoglobulin concentration}

Ig G and IgM revealed (Table 3) a synchronized highly significant increase $(p<0.01)$ in $G 1, G 2, G 4$, and $G 5$ compared to the controls with more significant increase $(p<0.01)$ in $\mathrm{G} 4$ and $\mathrm{G} 1$ treated with SeNPS, respectively compared to other groups with no significant differences between $\mathrm{G} 1$ and $\mathrm{G} 2$. The overall average concentrations of $\lg G$ and $\lg \mathrm{M}$ were significantly higher $(p<0.01)$ in $\operatorname{Ross}^{\circledR 3} 308$ (Mean=1625.8 mg/dL) compared to Arbor Acres ${ }^{\circledR}$ broilers (Mean $=1607.1 \mathrm{mg} /$ $\mathrm{dL})$.

IgG showed a highly significant decrease $(p<0.01)$ on the 38 day old broilers compared to the 19 day old in $\mathrm{G} 1$ and $\mathrm{G} 2$, no significant differences between the two slaughter times in G6, and a highly significant increase $(p<0.01)$ on the 38 day old broilers compared to the 19 day old in G3, G4 and G5 (Table 3). Meanwhile, IgM revealed a highly significant decrease $(p<0.01)$ on the 38 day old broilers compared to the 19 day old in $\mathrm{G} 1$ and $\mathrm{G} 2$, and a highly significant increase $(p<0.01)$ on the 38 day old broilers compared to the 19 day old in G3, G4, G5, and G6 (Table 3).

\section{Antioxidant profile}

TAC (Table 3 ) revealed a highly significant increase $(p<0.01)$ in $\mathrm{G} 1, \mathrm{G} 4, \mathrm{G} 5, \mathrm{G} 6, \mathrm{G} 3$, and $\mathrm{G} 2$, respectively. MDA showed a highly significant increase $(p<0.01)$ in G4, G1, G5, G2, G3, and G6, respectively with no significant differences between Arbor Acres $^{\circledR} \mathrm{G} 3$ and Ross ${ }^{\circledR 3} 08$ G6 controls. SOD revealed a highly significant increase $(p<0.01)$ in $G 1$ and $G 4$ over the other treated and control groups with no significant differences between $\mathrm{G} 1$ and $\mathrm{G} 4$ and between $\mathrm{G} 2, \mathrm{G} 3$, G5, and G6. Generally, TAC, MDA, and SOD revealed a highly significant increase $(p<0.01)$ in $\operatorname{Ross}^{\circledR 3} 308$ (Means= $1.64 \mathrm{mM} / \mathrm{L}, 15.6 \mathrm{nmol} / \mathrm{ml}$, and $259.8 \mathrm{U} / \mathrm{ml}$ respectively) than Arbor Acres $^{\circledR}$ broilers (Mean $=1.53$ $\mathrm{mM} / \mathrm{L}, 13.3 \mathrm{nmol} / \mathrm{ml}$, and $255.3 \mathrm{U} / \mathrm{ml}$ respectively) (Table 3).

TAC showed a highly significant decrease $(p<0.01)$ on the 38 day old broilers compared to

Table 3 - Immunoglobulin concentration (Mean \pm SE) and Antioxidant profile (Mean \pm SE) of different broiler breeds supplemented with selenium and green synthetized selenium nanoparticles.

\begin{tabular}{|c|c|c|c|c|c|c|}
\hline \multirow{2}{*}{$\begin{array}{l}\frac{\tilde{O}}{\bar{y}} \\
\text { o }\end{array}$} & \multirow{2}{*}{ 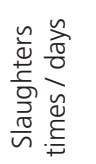 } & \multicolumn{2}{|c|}{ Immunoglobulin concentration } & \multicolumn{3}{|c|}{ Antioxidant enzymes } \\
\hline & & $\begin{array}{l}\mathrm{lgG} \\
\mathrm{mg} / \mathrm{dl}\end{array}$ & $\begin{array}{l}\mathrm{lgM} \\
\mathrm{mg} / \mathrm{dl}\end{array}$ & $\begin{array}{c}\text { TAC } \\
\mathrm{mM} / \mathrm{L}\end{array}$ & $\begin{array}{c}\mathrm{MDA} \\
\mathrm{nmol} / \mathrm{ml}\end{array}$ & $\begin{array}{l}\text { SOD } \\
\mathrm{U} / \mathrm{ml}\end{array}$ \\
\hline G1 & & $1739.9^{b} \pm 7.03$ & $414.8^{\mathrm{b}} \pm 3.14$ & $1.79^{\mathrm{a}} \pm 0.01$ & $21.3^{b} \pm 0.29$ & $260.5^{a} \pm 1.96$ \\
\hline G2 & & $1736.0^{b} \pm 6.65$ & $410.5^{b} \pm 2.97$ & $1.33^{f} \pm 0.01$ & $11.6^{d} \pm 0.27$ & $251.2^{b} \pm 1.85$ \\
\hline G3 & & $1345.5^{\mathrm{e}} \pm 7.03$ & $269.2^{\mathrm{e}} \pm 3.14$ & $1.49^{\mathrm{e}} \pm 0.01$ & $7.2^{\mathrm{e}} \pm 0.29$ & $254.3^{b} \pm 1.96$ \\
\hline G4 & & $1769.4^{\mathrm{a}} \pm 6.88$ & $425.0^{\mathrm{a}} \pm 3.07$ & $1.85^{b} \pm 0.01$ & $24.1^{\mathrm{a}} \pm 0.28$ & $267.8^{\mathrm{a}} \pm 1.92$ \\
\hline G5 & & $1729.9^{c} \pm 6.43$ & $399.0^{c} \pm 2.87$ & $1.58 \_ \pm 0.01$ & $14.7^{c} \pm 0.26$ & $257.2^{b} \pm 1.79$ \\
\hline G6 & & $1378.1^{\mathrm{d}} \pm 6.88$ & $301.5^{\mathrm{d}} \pm 3.07$ & $1.51^{\mathrm{d}} \pm 0.01$ & $7.9^{e} \pm 0.28$ & $254.5^{b} \pm 1.92$ \\
\hline$p$ value & & 0.000 & 0.001 & 0.000 & 0.000 & 0.000 \\
\hline \multicolumn{7}{|c|}{ Groups * Slaughter times } \\
\hline \multirow[t]{2}{*}{ G1 } & $19^{\text {th }}$ & $1841.6^{a} \pm 21.6$ & $487.4^{a} \pm 10.0$ & $1.66^{b} \pm 0.01$ & $18.8^{b} \pm 0.67$ & $236.9^{b} \pm 2.98$ \\
\hline & $38^{\text {th }}$ & $1638.2^{b} \pm 11.2$ & $342.2^{b} \pm 4.43$ & $1.93^{\mathrm{a}} \pm 0.01$ & $23.8^{\mathrm{a}} \pm 0.46$ & $284.1^{\mathrm{a}} \pm 0.78$ \\
\hline \multirow[t]{2}{*}{ G2 } & $19^{\text {th }}$ & $1829.2^{\mathrm{a}} \pm 10.3$ & $473.6^{a} \pm 5.68$ & $1.17^{b} \pm 0.00$ & $10.0^{b} \pm 0.33$ & $247.1^{b} \pm 2.10$ \\
\hline & $38^{\text {th }}$ & $1624.8^{b} \pm 7.06$ & $347.4^{b} \pm 2.05$ & $1.50^{\mathrm{a}} \pm 0.01$ & $13.2^{\mathrm{a}} \pm 0.31$ & $255.2^{\mathrm{a}} \pm 1.69$ \\
\hline \multirow[t]{2}{*}{ G3 } & $19^{\text {th }}$ & $1317.0^{b} \pm 11.1$ & $263.2^{b} \pm 4.04$ & $1.47^{\mathrm{a}} \pm 0.00$ & $6.9^{b} \pm 0.27$ & $259.6^{a} \pm 0.89$ \\
\hline & $38^{\text {th }}$ & $1374.0^{\mathrm{a}} \pm 7.09$ & $275.2^{\mathrm{a}} \pm 1.85$ & $1.51^{\mathrm{a}} \pm 0.01$ & $7.4^{\mathrm{a}} \pm 0.14$ & $248.9^{b} \pm 4.51$ \\
\hline \multirow[t]{2}{*}{ G4 } & $19^{\text {th }}$ & $1598.6^{b} \pm 10.2$ & $349.0^{b} \pm 11.6$ & $1.80^{b} \pm 0.02$ & $22.4^{b} \pm 0.53$ & $247.0^{b} \pm 5.25$ \\
\hline & $38^{\text {th }}$ & $1940.3^{a} \pm 9.73$ & $501.1^{a} \pm 1.03$ & $1.89^{a} \pm 0.01$ & $25.9^{a} \pm 0.62$ & $288.6^{a} \pm 0.34$ \\
\hline \multirow[t]{2}{*}{ G5 } & $19^{\text {th }}$ & $1579.0^{b} \pm 9.75$ & $337.6^{b} \pm 6.50$ & $1.54^{b} \pm 0.01$ & $12.4^{b} \pm 0.26$ & $248.2^{b} \pm 0.82$ \\
\hline & $38^{\text {th }}$ & $1880.8^{a} \pm 5.51$ & $460.4^{a} \pm 1.77$ & $1.61^{\mathrm{a}} \pm 0.01$ & $17.0^{\mathrm{a}} \pm 0.21$ & $266.2^{\mathrm{a}} \pm 1.91$ \\
\hline \multirow[t]{2}{*}{ G6 } & $19^{\text {th }}$ & $1378.6^{a} \pm 4.29$ & $294.0^{b} \pm 2.98$ & $1.52^{\mathrm{a}} \pm 0.00$ & $7.6^{a} \pm 0.12$ & $260.0^{a} \pm 1.13$ \\
\hline & $38^{\text {th }}$ & $1377.7^{a} \pm 3.32$ & $309.1^{\mathrm{a}} \pm 1.81$ & $1.50^{\mathrm{a}} \pm 0.03$ & $8.2^{\mathrm{a}} \pm 0.11$ & $249.1^{b} \pm 2.91$ \\
\hline$p$ value & & 0.003 & 0.042 & 0.000 & 0.000 & 0.000 \\
\hline
\end{tabular}

Means carrying different superscripts in the same column are significantly different at $(p \leq 0.05)$ or highly significantly different at $(p<0.01)$. Means carrying the same superscripts in the same column are non-significantly different at $(p<0.05)$.

$\mathrm{G} 1=$ Arbor Acres ${ }^{\circledR}$ with $1 \mathrm{~mL}$ nano-selenium/1L drinking water, $\mathrm{G} 2=$ Arbor Acres ${ }^{\circledR}$ with $1 \mathrm{~mL}$ selenium/1L drinking water), G3=Arbor Acres ${ }^{\circledR}$ control, $\mathrm{G} 4=$ Ross $^{\circledR} 308$ with $1 \mathrm{~mL}$ nano-selenium/1 L drinking water, $\mathrm{G} 5=$ Ross $^{\circledR} 308$ with $1 \mathrm{~mL}$ selenium $/ 1 \mathrm{~L}$ drinking water, and $\mathrm{G} 6=$ Ross ${ }^{\circledR} 308$ control.

Ig $\mathrm{G}=$ Immunoglobulin $\mathrm{G}$, IgM=Immunoglobulin M, TAC=Total Antioxidant capacity, MDA= Malondialdehyde, and SOD=Superoxide Dismutase, SE=Standard error. 
the 19 day old in G2, no significant differences between the two slaughter times in $\mathrm{G} 3$ and $\mathrm{G} 6$, and a highly significant increase $(p<0.01)$ on the 38 day old broilers compared to the 19 day old in G1, G4 and G5 (Table 3). MDA revealed a highly significant decrease $(p<0.01)$ on the 38 day old broilers compared to the 19 day old in G2, no significant differences between the two slaughter times in G6, and a highly significant increase $(p<0.01)$ on the 38 day old broilers compared to the 19 day old in G1, G3, G4 and G5 (Table 3). SOD showed a highly significant decrease $(p<0.01)$ on the 38 day old broilers compared to the 19 day old in $\mathrm{G} 3$ and $\mathrm{G} 6$, and a highly significant increase $(p<0.01)$ on the 38 day old broilers compared to the 19 day old in $\mathrm{G} 1$, G2, G4 and G5 (Table 3).

\section{Bacterial Counts}

TBC revealed a highly significant decrease $(p<0.01)$ in intestinal swabs of $\mathrm{G} 5$ compared to $\mathrm{G} 4$ Ross $^{\circledR} 308$ broilers, and in G1 compared to G2 Arbor Acres ${ }^{\circledR}$ broilers, with no significant differences between G3 and G6 control of the two breeds, and a highly significant decrease $(p<0.01)$ in TBC of breast muscles of $\mathrm{G} 1$ compared to $\mathrm{G} 2$ Arbor Acres ${ }^{\circledR}$ broilers, and a significant decrease $(p \leq 0.05)$ in $\mathrm{G} 4$ compared to $\mathrm{G} 5$ Ross ${ }^{\circledR 3} 308$ broilers (Table 4).

TEC (Table 4) showed no significant differences between $\mathrm{G} 1$ and $\mathrm{G} 2$ Arbor Acres ${ }^{\circledR}$ intestinal swabs and breast muscles, and between $\mathrm{G} 4$ and $\mathrm{G} 5$ Ross $^{\circledR} 308$ broilers intestinal swabs, a highly significant difference $(p<0.01)$ between $\mathrm{G} 4$ and $\mathrm{G} 5$ Ross $^{\circledR} 308$ broilers breast muscles. TBC and TEC of intestinal swabs and breast muscles revealed a highly significant decrease $(p<0.01)$ in $\mathrm{G} 4$ and $\mathrm{G} 5 \operatorname{Ross}^{\circledR} 308$ (Mean=4.1, 3.1, 2, and 1.0 CFU/mL respectively) compared to $\mathrm{G} 1$ and $\mathrm{G} 2$ Arbor Acres ${ }^{\circledR}$ broilers (Mean=4.5, 3.4, 2.8, and 1.4 CFU/mL respectively).

TBC of intestinal swabs showed no significant differences in $\mathrm{G} 4$, and a highly significant increase $(p<0.01)$ on the 38 day old broilers compared to the 19 day old in G1, G2, G3, G5 and G6 (Table 4). TBC of breast muscles showed a highly significant decrease $(p<0.01)$ on the 38 day old broilers compared to the 19 day old in $\mathrm{G} 3, \mathrm{G} 4$, and $\mathrm{G} 6$, and a highly significant increase $(p<0.01)$ on the 38 day old broilers compared to the 19 day old in $\mathrm{G} 1, \mathrm{G} 2$, and $\mathrm{G} 5$ (Table 4).TEC of intestinal swabs and breast muscles showed a highly significant increase on the 38 day old broilers compared to the 19 day old in all treated and control groups (Table 4).

\section{Productive performance}

WG/g revealed a significant difference $(p \leq 0.05)$ between $G 1$ and $G 2$ and between $G 4$ and $G 5$ with no significant differences between $\mathrm{G} 3$ and $\mathrm{G} 6$ controls (Table 5).WG also revealed a highly significant increase $(p<0.01)$ on the $5^{\text {th }}, 3^{\text {rd }}, 4^{\text {th }}, 2^{\text {nd }}$, and $1^{\text {st }}$ week of age respectively in $\mathrm{G} 1, \mathrm{G} 2$, and $\mathrm{G} 3$ Arbor $A$ cres $^{\circledR}$ broilers, at on the $4^{\text {th }}, 3^{\text {rd }}, 5^{\text {th }}, 2^{\text {nd }}$, and $1^{\text {st }}$ week of age respectively in G4 Ross ${ }^{\circledR} 308$ broilers, and at on the $5^{\text {th }}, 4^{\text {th }}, 3^{\text {rd }}$, $2^{\text {nd }}$, and $1^{\text {st }}$ week of age respectively in $\mathrm{G} 5$ and $\mathrm{G} 6$ Ross ${ }^{\circledR 3} 08$ broilers (Table 5).

$\mathrm{Fl} / \mathrm{g}$ (Table 5) showed a highly significant increase in G4 compared to G5 and G6 Ross ${ }^{\circledR 3} 308$ control, and no significant differences between $\mathrm{G} 1$ and $\mathrm{G} 2$ Arbor Acres ${ }^{\circledR}$ broilers with a highly significant difference between the last two groups and G3 Arbor Acres ${ }^{\circledR}$ control. FI revealed (Table 5) a highly significant increase $(p<0.01)$ on the $5^{\text {th }}, 4^{\text {th }}, 3^{\text {rd }}, 2^{\text {nd }}$, and $1^{\text {st }}$ week of age respectively in $\mathrm{G} 1, \mathrm{G} 2, \mathrm{G} 5$, and $\mathrm{G} 6$ broilers, and on the $4^{\text {th }}, 5^{\text {th }}, 3^{\text {rd }}, 2^{\text {nd }}$, and $1^{\text {st }}$ week of age respectively in G3 Arbor Acres ${ }^{\circledR}$ control and G4 Ross ${ }^{\circledR} 308$ broilers.

FCR\% showed a significant difference $(p<0.01)$ between $G 1$ and $G 2$ with no significant differences between $\mathrm{G} 2$ and $\mathrm{G} 3$ Arbor Acres $^{\circledR}$ control, and a highly significant difference $(p<0.01)$ between $\mathrm{G} 4$ and $G 5$ with no significant differences between $G 4$ and G6 Ross ${ }^{\circledR 3} 308$ control (Table 5). Meanwhile, on a weekly basis, FCR revealed a highly significant increase $(p<0.01)$ on the $4^{\text {th }}, 3^{\text {rd }}, 2^{\text {nd }}, 1^{\text {st }}$, and $5^{\text {th }}$ week of age respectively in $G 1$, a highly significant increase $(p<0.01)$ on the $4^{\text {th }}, 3^{\text {rd }}, 2^{\text {nd }}, 5^{\text {th }}$, and $1^{\text {st }}$ week of age respectively with no significant differences between $2^{\text {nd }}$ and $3^{\text {rd }}$ weeks in $G 2$, a highly significant increase $(p<0.01)$ on the $4^{\text {th }}, 5^{\text {th }}, 2^{\text {nd }}, 3^{\text {rd }}$, and $1^{\text {st }}$ week of age respectively with no significant differences between the $1^{\text {st }}$ and $3^{\text {rd }}$ weeks in $\mathrm{G} 3$, a highly significant increase $(p<0.01)$ on the $5^{\text {th }}, 2^{\text {nd }}, 3^{\text {rd }}, 4^{\text {th }}$, and $1^{\text {st }}$ week of age respectively in G4, a highly significant increase $(p<0.01)$ on the $4^{\text {th }}$, $5^{\text {th }}, 3^{\text {rd }}, 2^{\text {nd }}$, and $1^{\text {st }}$ week of age respectively with no significant differences between the $1^{\text {st }}$ and $2^{\text {nd }}$ weeks in $\mathrm{G} 5$, and a highly significant increase $(p<0.01)$ on the $4^{\text {th }}, 3^{\text {rd }}, 5^{\text {th }}, 1^{\text {st }}$, and $2^{\text {nd }}$ week of age respectively with no significant difference between the $3^{\text {rd }}$ and $5^{\text {th }}$ weeks in G6.

Pl showed a significant difference $(p<0.01)$ between G1, G2 and G3 Arbor Acres ${ }^{\circledR}$ broilers, and a highly significant differences $(p<0.01)$ between $\mathrm{G} 4$ and $\mathrm{G} 5$ with significant differences $(p \leq 0.05)$ between $\mathrm{G} 4$ and G6 Ross ${ }^{\circledR 3} 38$ control. PI (Table 5) showed also a highly significant increase $(p<0.01)$ on the $5^{\text {th }}, 4^{\text {th }}, 3^{\text {rd }}, 2^{\text {nd }}$, and $1^{\text {st }}$ week of age respectively with no significant 
Table 4 - Logarithm bacterial load (Mean \pm SE) in intestine and breast muscles of different broiler breeds supplemented with selenium and green synthetized selenium nanoparticles.

\begin{tabular}{|c|c|c|c|c|c|}
\hline \multirow{2}{*}{$\begin{array}{l}\tilde{\frac{\Omega}{3}} \\
\frac{0}{0}\end{array}$} & \multirow{2}{*}{ 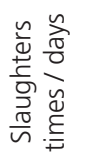 } & \multicolumn{2}{|c|}{ Intestinal Swabs } & \multicolumn{2}{|c|}{ Breast Muscles } \\
\hline & & Log. TBC CFU/mL & Log. TEC CFU/mL & Log. TBC CFU/mL & Log. TEC CFU/mL \\
\hline G1 & & $5.0^{b} \pm 0.049$ & $3.3^{a} \pm 0.127$ & $3.5^{b} \pm 0.043$ & $1.7^{\mathrm{a}} \pm 0.072$ \\
\hline G2 & & $5.3^{a} \pm 0.047$ & $3.0^{\mathrm{a}} \pm 0.120$ & $3.9^{\mathrm{a}} \pm 0.040$ & $1.8^{\mathrm{a}} \pm 0.068$ \\
\hline G3 & & $3.4^{e} \pm 0.049$ & $2.2^{b} \pm 0.127$ & $3.0^{d} \pm 0.043$ & $0.9^{c} \pm 0.072$ \\
\hline G4 & & $4.3^{d} \pm 0.048$ & $1.9^{\mathrm{b}} \pm 0.124$ & $3.1^{\mathrm{cd}} \pm 0.042$ & $0.9 c \pm 0.071$ \\
\hline G5 & & $4.7^{c} \pm 0.045$ & $2.1^{b} \pm 0.116$ & $3.2^{c} \pm 0.039$ & $1.4^{b} \pm 0.066$ \\
\hline G6 & & $3.4^{e} \pm 0.048$ & $2.0^{b} \pm 0.124$ & $3.1^{\mathrm{cd}} \pm 0.042$ & $0.9^{c} \pm 0.071$ \\
\hline$p$ value & & 0.000 & 0.000 & 0.000 & 0.000 \\
\hline \multicolumn{6}{|c|}{ Groups * Slaughter times } \\
\hline \multirow[t]{2}{*}{ G1 } & $19^{\text {th }}$ & $4.4^{b} \pm 0.25$ & $2.4^{b} \pm 0.61$ & $3.0^{b} \pm 0.09$ & $1.3^{b} \pm 0.36$ \\
\hline & $38^{\text {th }}$ & $5.7^{\mathrm{a}} \pm 0.02$ & $4.2^{\mathrm{a}} \pm 0.03$ & $4.1^{\mathrm{a}} \pm 0.02$ & $2.1^{\mathrm{a}} \pm 0.03$ \\
\hline \multirow[t]{2}{*}{ G2 } & $19^{\text {th }}$ & $3.9^{b} \pm 0.07$ & $1.4^{b} \pm 0.60$ & $3.2^{b} \pm 0.09$ & $0.5^{b} \pm 0.31$ \\
\hline & $38^{\text {th }}$ & $6.6^{a} \pm 0.01$ & $4.5^{a} \pm 0.02$ & $4.6^{a} \pm 0.02$ & $3.1^{\mathrm{a}} \pm 0.02$ \\
\hline \multirow[t]{2}{*}{ G3 } & $19^{\text {th }}$ & $3.1^{b} \pm 0.05$ & $0.0^{b} \pm 0.00$ & $3.5^{a} \pm 0.21$ & $0.0^{b} \pm 0.00$ \\
\hline & $38^{\text {th }}$ & $3.8^{\mathrm{a}} \pm 0.03$ & $4.4^{\mathrm{a}} \pm 0.01$ & $2.5^{b} \pm 0.04$ & $1.8^{\mathrm{a}} \pm 0.01$ \\
\hline \multirow[t]{2}{*}{ G4 } & $19^{\text {th }}$ & $4.3^{\mathrm{a}} \pm 0.14$ & $0.0^{b} \pm 0.00$ & $3.2^{\mathrm{a}} \pm 0.06$ & $0.0^{b} \pm 0.00$ \\
\hline & $38^{\text {th }}$ & $4.3^{a} \pm 0.02$ & $3.9 a \pm 0.02$ & $3.0^{b} \pm 0.00$ & $1.8^{\mathrm{a}} \pm 0.01$ \\
\hline \multirow[t]{2}{*}{ G5 } & $19^{\text {th }}$ & $3.9^{b} \pm 0.07$ & $0.0^{b} \pm 0.00$ & $3.1^{b} \pm 0.04$ & $0.0^{b} \pm 0.00$ \\
\hline & $38^{\text {th }}$ & $5.5^{a} \pm 0.01$ & $4.3^{a} \pm 0.03$ & $3.3^{a} \pm 0.01$ & $2.7^{\mathrm{a}} \pm 0.01$ \\
\hline \multirow[t]{2}{*}{ G6 } & $19^{\text {th }}$ & $3.0^{b} \pm 0.04$ & $0.0^{b} \pm 0.00$ & $3.3^{a} \pm 0.02$ & $0.0^{b} \pm 0.00$ \\
\hline & $38^{\text {th }}$ & $3.8^{\mathrm{a}} \pm 0.01$ & $4.0^{\mathrm{a}} \pm 0.00$ & $2.8^{b} \pm 0.02$ & $1.8^{\mathrm{a}} \pm 0.01$ \\
\hline$p$ value & & 0.000 & 0.000 & 0.000 & 0.000 \\
\hline
\end{tabular}

Means carrying different superscripts in the same column are significantly different at $(p \leq 0.05)$ or highly significantly different at $(p<0.01)$. Means carrying the same superscripts in the same column are non-significantly different at $(p<0.05)$.

G1=Arbor Acres ${ }^{\circledast}$ with $1 \mathrm{~mL}$ nano-selenium/1L drinking water, G2=Arbor Acres ${ }^{\circledast}$ with $1 \mathrm{~mL}$ selenium/1L drinking water), G3=Arbor Acres ${ }^{\circledR}$ control, G4= Ross $^{\oplus} 308$ with $1 \mathrm{~mL}$ nano-selenium $/ 1 \mathrm{~L}$ drinking water, $\mathrm{G} 5=$ Ross $^{\oplus 3} 308$ with $1 \mathrm{~mL}$ selenium/1 $\mathrm{L}$ drinking water, and $\mathrm{G} 6=\mathrm{Ross}^{\circledR} 308$ control.

$\mathrm{TBC}=$ Total Bacterial Count, TEC=Total Enterobacteriaceae Count, $\mathrm{CFU}=$ Colony Forming Unit, $\mathrm{SE}=$ Standard error

difference between the $3^{\text {rd }}$ and $4^{\text {th }}$ weeks in $\mathrm{G} 1$, a highly significant increase $(p<0.01)$ on the $5^{\text {th }}, 3^{\text {rd }}, 4^{\text {th }}, 2^{\text {nd }}$, and $1^{\text {st }}$ week of age respectively in $\mathrm{G} 2$, a highly significant increase $(p<0.01)$ on the $5^{\text {th }}, 4^{\text {th }}, 3^{\text {rd }}, 2^{\text {nd }}$, and $1^{\text {st }}$ week of age respectively with no significant difference between the $4^{\text {th }}$ and $5^{\text {th }}$ weeks and between the $2^{\text {nd }}$ and $3^{\text {rd }}$ in G3, a highly significant increase $(p<0.01)$ on the $4^{\text {th }}$, $5^{\text {th }}, 3^{\text {rd }}, 2^{\text {nd }}$, and $1^{\text {st }}$ week of age respectively in $\mathrm{G} 4$, and a highly significant increase $(p<0.01)$ on the $5^{\text {th }}, 4^{\text {th }}$, $3^{\text {rd }}, 2^{\text {nd }}$, and $1^{\text {st }}$ week of age respectively in $\mathrm{G} 5$ and $\mathrm{G} 6$.

The overall means of $\mathrm{WG}, \mathrm{Fl}$, and $\mathrm{Pl}$ indicated a highly significant increase $(p<0.01)$ in Arbor Acres ${ }^{\circledR}$ (380.08g, 589.1g, and 5.98) compared to Ross ${ }^{\circledR 3} 308$ broilers $(378.88 \mathrm{~g}, 639.0 \mathrm{~g}$, and 5.67 ) respectively, meanwhile FCR revealed a highly significant increase $(p<0.01)$ in $\operatorname{Ross}^{\circledR} 308(1.67 \%)$ compared to Arbor Acres ${ }^{\circledR}$ broilers (1.62\%).

\section{Histopathological architecture examination}

Liver photomicrographs revealed in G1 (Figure 3a) and G2 (Figure 3b) thickening of liver capsule due to fibrinous exudation and mononuclear cell infiltration, hepatic cells showed degeneration with mild vacuolation of cytoplasm and mononuclear cell infiltration, as well as, mild hemorrhage in $\mathrm{G} 2$ broilers (Figure 3b). Meanwhile, the liver of G4 (Figure 3d) showed congestion of central vein, the hepatocytes showed mild degeneration, cytoplasmic vacuolation and mononuclear cell infiltration. The liver of $\mathrm{G} 5$ (Figure 3e) showed mild perihepatitis due to fibrinous exudation and mononuclear cell infiltration, hepatic cells showed mild degeneration, mild hemorrhage and mononuclear cell infiltration compared to Arbor Acres ${ }^{\circledR}$ control (Figure 3c) and Ross $^{\circledR 3} 308$ control (Figure 3f).

Heart examination showed moderate fibrinous pericarditis which extend to myocardium resulting in myocardial degeneration with cytoplasmic vacuolation and mononuclear cell infiltration in G1 as revealed (Figure $4 a$ ) and $\mathrm{G} 2$ side by side to the presence of congestion and mild hemorrhage of cardiac muscle in G2 only (Figure 4b) compared 
Table 5 - Performance indices (Mean \pm SE) of different broiler breeds supplemented with selenium and green synthetized selenium nanoparticles.

\begin{tabular}{|c|c|c|c|c|c|}
\hline Groups & Age / wk & WG / g & $\mathrm{Fl} / \mathrm{g}$ & FCR \% & $\mathrm{Pl}$ \\
\hline G1 & & $384.42^{\mathrm{ab}} \pm 9.53$ & $608.41^{c} \pm 0.002$ & $1.72^{\mathrm{ab}} \pm$ & $5.72^{\mathrm{bcd}} \pm 0.11$ \\
\hline G2 & & $393.70^{a} \pm 9.91$ & $604.82^{c} \pm 0.001$ & $1.58^{b c} \pm$ & $6.19^{a b} \pm 0.19$ \\
\hline G3 & & $362.12^{b} \pm 8.86$ & $554.26^{d} \pm 0.001$ & $1.57^{b c_{ \pm}}$ & $6.03^{\mathrm{abc}} \pm 0.23$ \\
\hline G4 & & $399.68^{a} \pm 9.12$ & $720.69^{\mathrm{a}} \pm 0.001$ & $1.83^{\mathrm{a}} \pm$ & $5.48^{\mathrm{cd}} \pm 0.23$ \\
\hline G5 & & $373.62^{\mathrm{ab}} \pm 7.12$ & $557.47^{d} \pm 0.002$ & $1.45^{c} \pm$ & $6.34^{\mathrm{a}} \pm 0.15$ \\
\hline G6 & & $363.34^{b} \pm 8.21$ & $638.89^{b} \pm 0.001$ & $1.75^{\mathrm{a}} \pm$ & $5.19^{d} \pm 0.18$ \\
\hline$p$ value & & 0.021 & 0.000 & 0.069 & 0.000 \\
\hline \multicolumn{6}{|c|}{ Groups*Age of birds } \\
\hline \multirow{5}{*}{ G1 } & $1^{\text {st }}$ & $74.3^{e} \pm 6.87$ & $104.8^{e} \pm 0.001$ & $1.58 \pm 0.22$ & $0.90^{\mathrm{d}} \pm 0.11$ \\
\hline & $2^{\text {nd }}$ & $175.6^{d} \pm 18.28$ & $308.6^{d} \pm 0.001$ & $1.97^{b} \pm 0.24$ & $1.77^{\complement} \pm 0.26$ \\
\hline & $3^{\text {rd }}$ & $497.9^{b} \pm 36.17$ & $711.9^{c} \pm 0.000$ & $1.49^{\mathrm{d}} \pm 0.10$ & $5.67^{b} \pm 0.60$ \\
\hline & $4^{\text {th }}$ & $425.6^{c} \pm 28.60$ & $894.4^{b} \pm 0.001$ & $2.20^{\mathrm{a}} \pm 0.16$ & $5.81^{b} \pm 0.40$ \\
\hline & $5^{\text {th }}$ & $748.7^{a} \pm 15.86$ & $1022.2^{\mathrm{a}} \pm 0.000$ & $1.37 \mathrm{e} \pm 0.02$ & $14.43^{\mathrm{a}} \pm 0.36$ \\
\hline \multirow{5}{*}{ G2 } & $1^{\text {st }}$ & $96.1^{\mathrm{e}} \pm 6.46$ & $110.4^{e} \pm 0.001$ & $1.19^{d} \pm 0.06$ & $1.27^{e} \pm 0.15$ \\
\hline & $2^{\text {nd }}$ & $245.1^{\mathrm{d}} \pm 19.57$ & $323.6^{d} \pm 0.001$ & $1.40^{\mathrm{b}} \pm 0.12$ & $3.01^{d} \pm 0.35$ \\
\hline & $3^{\text {rd }}$ & $501.2^{b} \pm 17.98$ & $706.9^{c} \pm 0.000$ & $1.42^{b} \pm 0.05$ & $6.29^{b} \pm 0.26$ \\
\hline & $4^{\text {th }}$ & $351.8^{c} \pm 19.71$ & $862.5^{b} \pm 0.001$ & $2.53^{\mathrm{a}} \pm 0.16$ & $5.07 \_ \pm 0.32$ \\
\hline & $5^{\text {th }}$ & $774.3^{\mathrm{a}} \pm 28.81$ & $1020.5^{\mathrm{a}} \pm 0.001$ & $1.33^{c} \pm 0.04$ & $15.32^{a} \pm 0.73$ \\
\hline \multirow{5}{*}{ G3 } & $1^{\text {st }}$ & $90.3^{\mathrm{e}} \pm 3.38$ & $119.5^{\mathrm{e}} \pm 0.000$ & $1.33^{\mathrm{d}} \pm 0.04$ & $1.03^{c} \pm 0.06$ \\
\hline & $2^{\text {nd }}$ & $247.5^{d} \pm 10.22$ & $365.5^{d} \pm 0.001$ & $1.49^{c} \pm 0.05$ & $2.61^{c} \pm 0.17$ \\
\hline & $3^{\text {rd }}$ & $519.2^{b} \pm 25.03$ & $685.0^{`} \pm 0.001$ & $1.35^{\mathrm{d}} \pm 0.07$ & $6.90^{\mathrm{b}} \pm 0.48$ \\
\hline & $4^{\text {th }}$ & $409.4^{c} \pm 29.32$ & $812.6^{a} \pm 0.001$ & $2.07^{\mathrm{a}} \pm 0.14$ & $6.62^{b} \pm 0.51$ \\
\hline & $5^{\text {th }}$ & $544.2^{\mathrm{a}} \pm 42.41$ & $788.5^{b} \pm 0.001$ & $1.59^{b} \pm 0.22$ & $12.98^{a} \pm 1.15$ \\
\hline \multirow{5}{*}{ G4 } & $1^{\text {st }}$ & $86.8^{e} \pm 6.86$ & $115.8^{e} \pm 0.001$ & $1.42^{\mathrm{e}} \pm 0.13$ & $1.03^{e} \pm 0.12$ \\
\hline & $2^{\text {nd }}$ & $248.6^{d} \pm 12.87$ & $423.3^{d} \pm 0.000$ & $1.75^{\mathrm{b}} \pm 0.11$ & $2.26^{\mathrm{d}} \pm 0.17$ \\
\hline & $3^{\text {rd }}$ & $553.9^{b} \pm 20.88$ & $901.2^{c} \pm 0.001$ & $1.64^{c} \pm 0.06$ & $5.76^{c} \pm 0.29$ \\
\hline & $4^{\text {th }}$ & $706.5^{\mathrm{a}} \pm 13.86$ & $1086.1^{\mathrm{a}} \pm 0.002$ & $1.54^{\mathrm{d}} \pm 0.03$ & $10.68^{a} \pm 0.28$ \\
\hline & $5^{\text {th }}$ & $402.6^{c} \pm 27.30$ & $1076.9^{b} \pm 0.000$ & $2.78^{\mathrm{a}} \pm 0.18$ & $7.68^{b} \pm 0.60$ \\
\hline \multirow{5}{*}{ G5 } & $1^{\text {st }}$ & $103.6^{e} \pm 3.72$ & $125.8^{\mathrm{e}} \pm 0.000$ & $1.22^{\mathrm{d}} \pm 0.04$ & $1.23^{\mathrm{e}} \pm 0.07$ \\
\hline & $2^{\text {nd }}$ & $286.9^{d} \pm 18.00$ & $361.8 d \pm 0.001$ & $1.30^{d} \pm 0.07$ & $3.53^{d} \pm 0.5$ \\
\hline & $3^{\text {rd }}$ & $468.8^{c} \pm 9.95$ & $697.9^{c} \pm 0.001$ & $1.499^{c} \pm 0.03$ & $6.08^{c} \pm 0.19$ \\
\hline & $4^{\text {th }}$ & $474.2^{b} \pm 19.87$ & $780.3^{b} \pm 0.001$ & $1.67^{\mathrm{a}} \pm 0.07$ & $8.40^{b} \pm 0.40$ \\
\hline & $5^{\text {th }}$ & $534.6^{a} \pm 19.11$ & $821.4^{\mathrm{a}} \pm 0.001$ & $1.55^{\mathrm{b}} \pm 0.06$ & $12.47^{a} \pm 0.51$ \\
\hline \multirow{5}{*}{ G6 } & $1^{\text {st }}$ & $85.6^{e} \pm 5.86$ & $131.2^{\mathrm{e}} \pm 0.001$ & $1.60^{\complement} \pm 0.11$ & $0.88^{e} \pm 0.10$ \\
\hline & $2^{\text {nd }}$ & $258.3^{d} \pm 11.36$ & $363.7^{\mathrm{d}} \pm 0.002$ & $1.43^{\mathrm{d}} \pm 0.06$ & $2.81^{\mathrm{d}} \pm 0.17$ \\
\hline & $3^{\text {rd }}$ & $474.6 \pm 37.10$ & $785.8^{\complement} \pm 0.002$ & $1.82^{b} \pm 0.25$ & $5.38^{c} \pm 0.55$ \\
\hline & $4^{\text {th }}$ & $484.0^{b} \pm 25.31$ & $955.3^{b} \pm 0.000$ & $2.01^{\mathrm{a}} \pm 0.09$ & $6.84^{b} \pm 0.34$ \\
\hline & $5^{\text {th }}$ & $514.2^{\mathrm{a}} \pm 23.23$ & $958.3^{a} \pm 0.001$ & $1.89^{b} \pm 0.08$ & $10.03^{a} \pm 0.50$ \\
\hline$p$ value & & 0.000 & 0.000 & 0.002 & 0.000 \\
\hline
\end{tabular}

Means carrying different superscripts in the same column are significantly different at $(p \leq 0.05)$ or highly significantly different at $(p<0.01)$. Means carrying the same superscripts in the same column are non-significantly different at $(p<0.05)$.

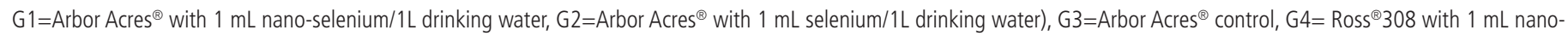
-selenium $/ 1 \mathrm{~L}$ drinking water, $\mathrm{G} 5=$ Ross $^{\circledR 3} 308$ with $1 \mathrm{~mL}$ selenium $/ 1 \mathrm{~L}$ drinking water, and $\mathrm{G} 6=$ Ross ${ }^{\circledR 3} 308$ control.

WG=Weight Gain, Fl=Feed Intake, FCR=Feed Conversion Ratio, and PI=Performance Index, SE=Standard error.

to G3 control (Figure 4c). G4 Ross ${ }^{\circledR 308}$ broilers revealed (Figure $4 \mathrm{~d}$ ) mild pericarditis, mild myocardial degeneration with mild congestion, cytoplasmic vacuolation and mononuclear cell infiltration. Heart of G5 (Figure 4e) showed mild fibrinous pericarditis, myocardial degeneration with mild hemorrhage cytoplasmic vacuolation and mononuclear cell infiltration compared to G6 (Figure 4f).
The spleen of $\mathrm{G} 1$ and $\mathrm{G} 2$ (Figures $5 a$ and $5 b$ ) revealed congestion of splenic sinus, mild hemorrhage with hemosedrosis, and moderate lymphoid depletion compared to G3 (Figure 5c). Meanwhile, G4 in Figure $5 d$ revealed no pathological changes, and $\mathrm{G} 5$ showed moderate lymphoid depletion of spleen and hemorrhage (Figure 5e) compared to the normal picture in Ross ${ }^{\circledR 3} 308$ broilers of G6 (Figure 5f). 
Ali AA, Soliman ES, Hamad RT, El-Borad OM, Hassan RA, Helal MS

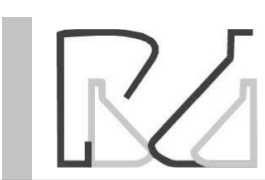

\section{Preventive, Behavioral, Productive, and Tissue Modification using Green Synthesized Selenium Nanoparticles in the Drinking Water of Two Broiler Breeds under Microbial Stress}
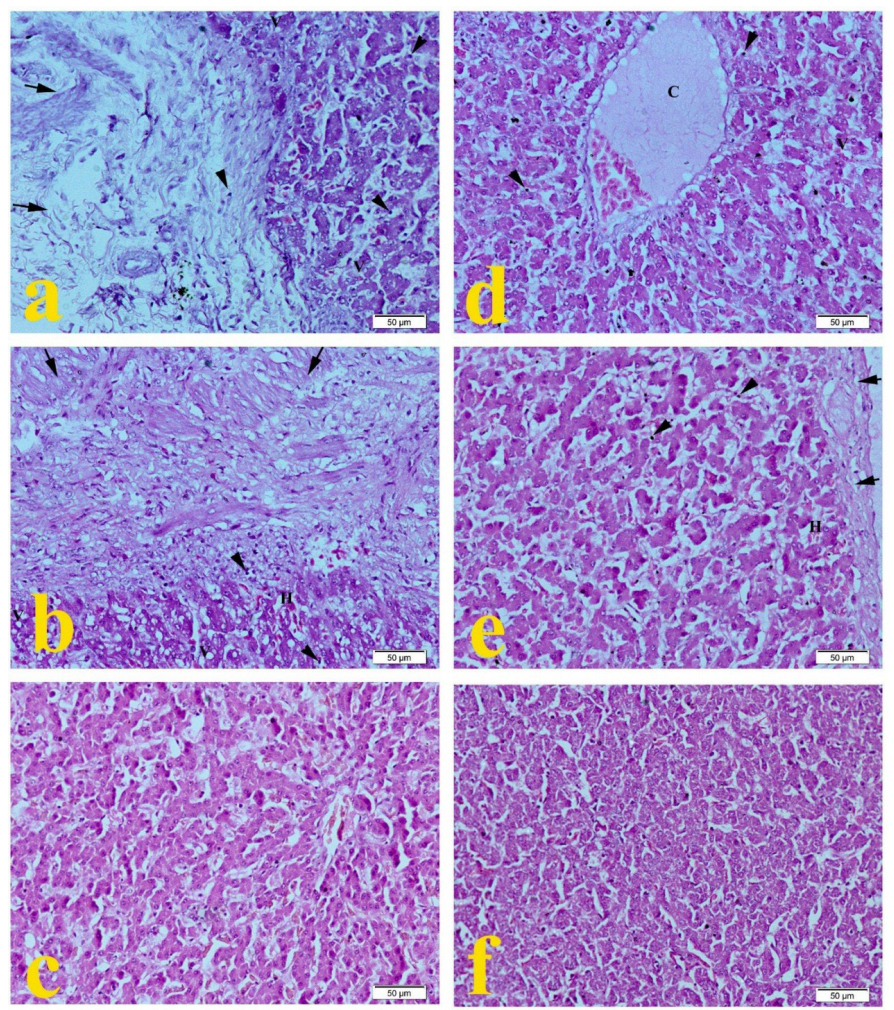

Figure 3 - Representative photomicrographs of liver histopathology (20x): (a) Liver of Arbor Acres ${ }^{\circledR}$ broilers supplemented with $1 \mathrm{~mL}$ nano-selenium/1L drinking water showing severe fibrosis (arrow), mononuclear cell infiltration (arrow head), vaculation of hepatocytes cytoplasm (V). (b) Liver of Arbor Acres ${ }^{\circledR}$ broilers supplemented with 1 $\mathrm{mL}$ selenium/1L drinking water. (c) Liver of control Arbor Acres ${ }^{\circledR}$ broilers. (d) Liver of Ross ${ }^{\circledR} 308$ broilers supplemented with $1 \mathrm{~mL}$ nano-selenium/1L drinking water. (e) Liver of Ross $® 308$ broilers supplemented with $1 \mathrm{~mL}$ selenium/1L drinking water. (f) Liver of control Ross $® 308$ broilers. H\&E. Bar $50 \mu \mathrm{m}$.

Histopathological examination of the bursa of fabricious revealed severe thickening and hyperplasia of follicular epithelium with severe depletion of lymphoid follicles which are replaced by edematous fluid and fibrous tissue in G1 (Figure 6a). Severe hyperplasia of follicular epithelium with severe lymphoid depletion of lymphoid follicles were revealed in G2 (Figure 6b) in comparison to $\mathrm{G} 3$ control (Figure 6c). On the other hand, G4 in Figure 6d showed normal histologic structure of lymphoid follicles with increased interfollicular fibrosis, G5 (Figure 6e) showed moderate lymphoid depletion with mild necrosis of lymphoid follicles with increased interfollicular fibrosis compared to G6 control (Figure 6f).

Photomicrographs of thymus showed moderate depletion of lymphocytes in the cortex and medulla in G1 (Figure 7a), moderate to severe depletion of the lymphocytes in the cortex and medulla in G2 (Figure 7b) in relation to normal architecture in G3 (Figure 7c). G4 (Figure 7d) showed normal histologic structure of both the cortex and medulla as those in G6 Ross ${ }^{\circledR 3} 08$ broilers (Figure 7f), while G5 showed moderate
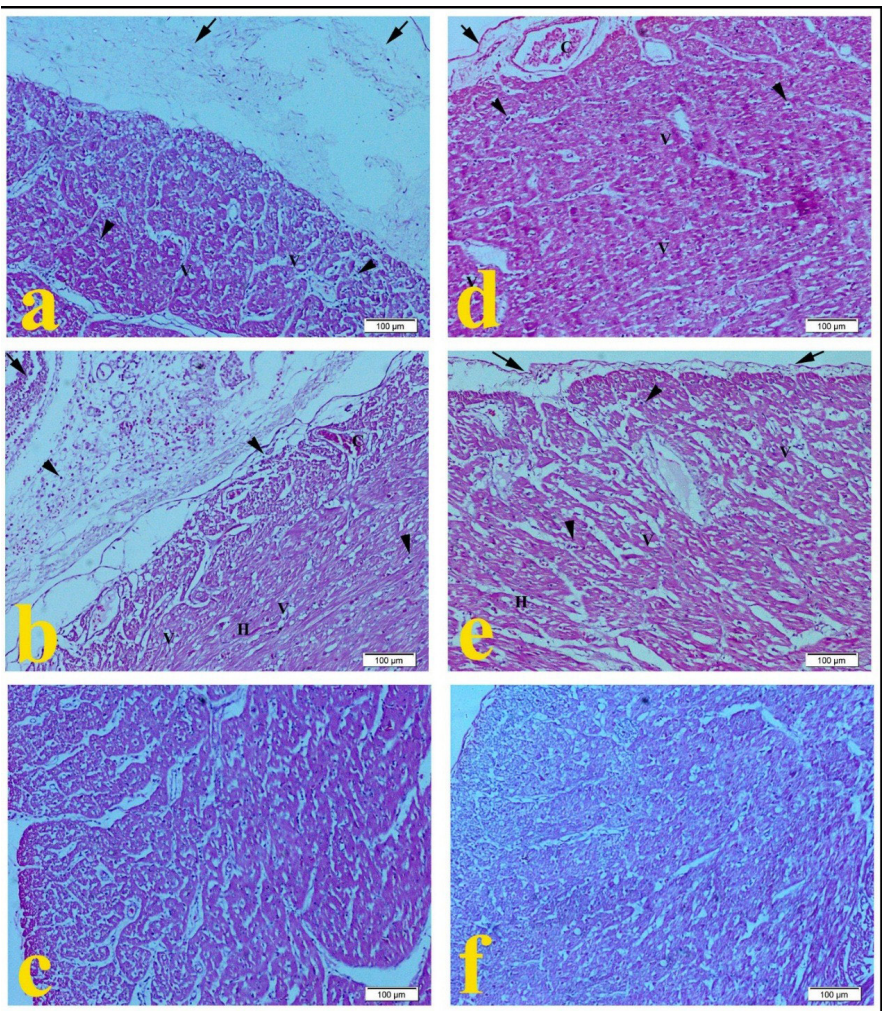

Figure 4 - Representative photomicrographs of Heart histopathology (10x): (a) Heart of Arbor Acres $^{\circledR}$ broilers supplemented with $1 \mathrm{~mL}$ nano-selenium/1L drinking water showing fibrinous pericarditis (arrow), mononuclear cell infiltration (arrow head), vaculation of cytoplasm (V). (b) Heart of Arbor Acres ${ }^{\circledR}$ broilers supplemented with 1 $\mathrm{mL}$ selenium/1L drinking water. (c) Heart of control Arbor Acres ${ }^{\circledR}$ broilers. (d) Heart of Ross ${ }^{\circledR} 308$ broilers supplemented with $1 \mathrm{~mL}$ nano-selenium/1L drinking water. (e) Heart of Ross ${ }^{\circledR} 308$ broilers supplemented with $1 \mathrm{~mL}$ selenium/1L drinking water. (f) Heart of control Ross ${ }^{\circledR} 308$ broilers. H\&E. Bar $100 \mu \mathrm{m}$.

depletion of lymphocytes in the cortex and medulla (Figure 7e).

\section{DISCUSSION}

Selenium has been considered an essential element required for multiple functions in broiler as productivity, growth, fertility, and prophylaxis via increasing immunoglobulin concentrations as reported by Del Puerto et al. (2017). Based on the European Union recommendation (2004) selenium has to be supplemented at $0.5 \mathrm{mg} / \mathrm{kg}$ dry matter to contribute a notable enhancement of performance, immunity, and antioxidant activity.

Broiler's behavior attested to an improvement in Ross $^{\circledR 3} 308$ and Arbor Acres ${ }^{\circledR}$ broilers supplemented with $1 \mathrm{~mL}$ SeNPS (100 mg/1 L) as represented by higher feeding frequency, drinking duration and frequency, walking duration and frequency, and resting frequency. These finding were in agreement with Omidi et al. (2018) who reported that reduced selenium levels may create oxidative stress, contributing to an increase in the risk of behavioral disturbances. In 
Ali AA, Soliman ES, Hamad RT, El-Borad OM, Hassan RA, Helal MS

\section{Preventive, Behavioral, Productive, and Tissue Modification using Green Synthesized Selenium Nanoparticles in the Drinking Water of Two Broiler Breeds under Microbial Stress}
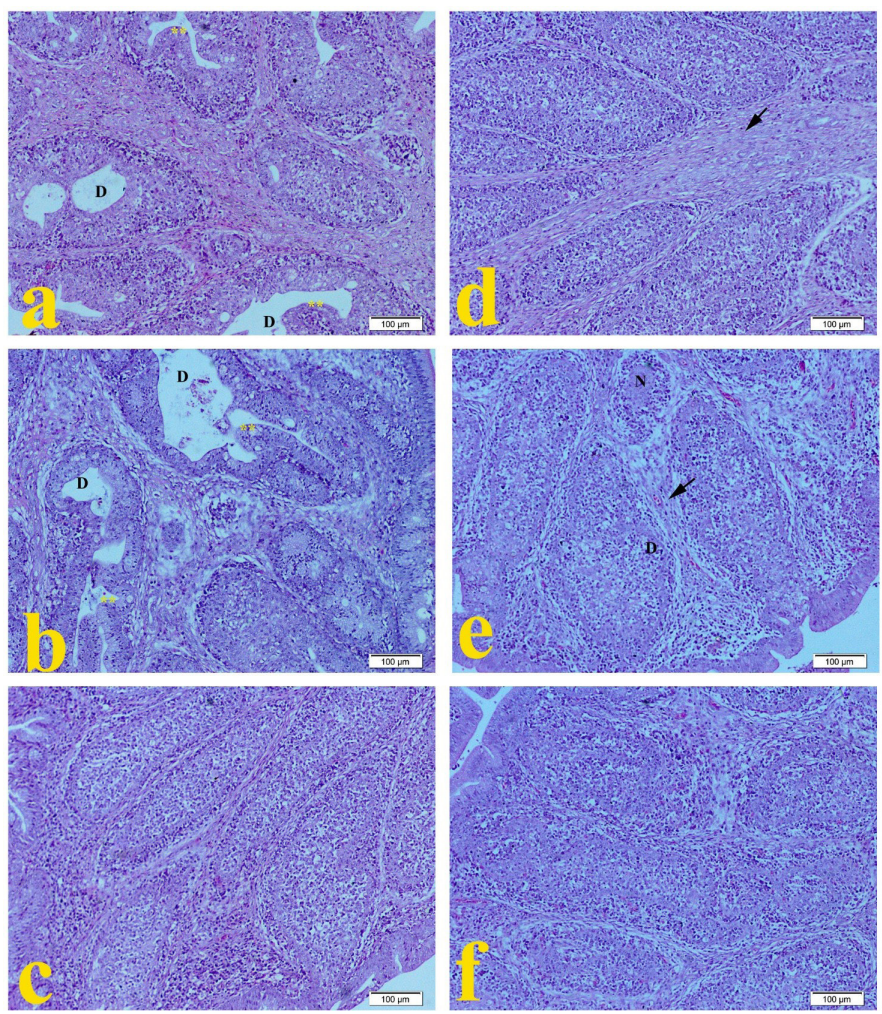

Figure 6 - Representative photomicrographs of Bursa histopathology (10x): (a) Bursa of Arbor Acres ${ }^{\circledR}$ broilers supplemented with $1 \mathrm{~mL}$ nano-selenium/1L drinking water showing depletion of lymphocytes (D), hyperplasia of follicular epithelium (**). (b) Bursa of Arbor Acres ${ }^{\circledR}$ broilers supplemented with $1 \mathrm{~mL}$ selenium/1L drinking water. (c) Bursa of control Arbor Acres ${ }^{\circledR}$ broilers. (d) Bursa of Ross ${ }^{\circledR} 308$ broilers supplemented with $1 \mathrm{~mL}$ nano-selenium/1L drinking water. (e) Bursa of Ross ${ }^{\circledR 3} 308$ broilers supplemented with 1 $\mathrm{mL}$ selenium/1L drinking water. (f) Bursa of control Ross ${ }^{\circledR} 308$ broilers. H\&E. Bar $100 \mu \mathrm{m}$.

and IgM concentrations in Ross ${ }^{\circledR} 308$ and Arbor Acres ${ }^{\circledR}$ broilers supplemented with $1 \mathrm{~mL}$ SeNPS $(100 \mathrm{mg} / 1 \mathrm{~L}$ ), with more significant increase in Ross ${ }^{\circledR 3} 38$ broilers. The increase was significantly notable on the $19^{\text {th }}$ day in treated Ross ${ }^{\circledR} 308$ broilers and on the $38^{\text {th }}$ day in treated Arbor Acres ${ }^{\circledR}$ broilers. The results were in agreement to those of Ahmadi etal. (2018) who reported a significant increase in immunoglobulin of one-day old male Ross 308 chicks supplemented with $0.1,0.2,0.3,0.4$, and $0.5 \mathrm{mg} \mathrm{SeNPS} / \mathrm{kg}$ feed. Bakhshalinejad et al. (2018) were also in agreement with our results, they used 0.4 mg SeNPS/kg for 1200 one-day-old male Ross broilers and found an enhancement in IgG concentrations. Zamani Moghaddam et al. (2017) proved a significant increase of immunoglobulin in broilers supplemented with $0.3 \mathrm{mg}$ organic selenium $/ \mathrm{kg}$ feed.

Glutathione (GSH) and SOD are considered major antioxidant defenses against oxygen reducing metabolites. Meanwhile, TAC and MDA usually increased relevant to oxidative and environmental stressors of broilers (Gangadoo et al., 2018). Nanoselenium at rate of $1 \mathrm{~mL}(100 \mathrm{mg} / 1 \mathrm{~L})$ was able to a meliorate TAC, SOD, and MDA activity in the recent study to alleviate the impact of microbial stress induced 
Ali AA, Soliman ES, Hamad RT, El-Borad OM, Hassan RA, Helal MS
Preventive, Behavioral, Productive, and Tissue Modification using Green Synthesized Selenium Nanoparticles in the Drinking Water of Two Broiler Breeds under Microbial Stress
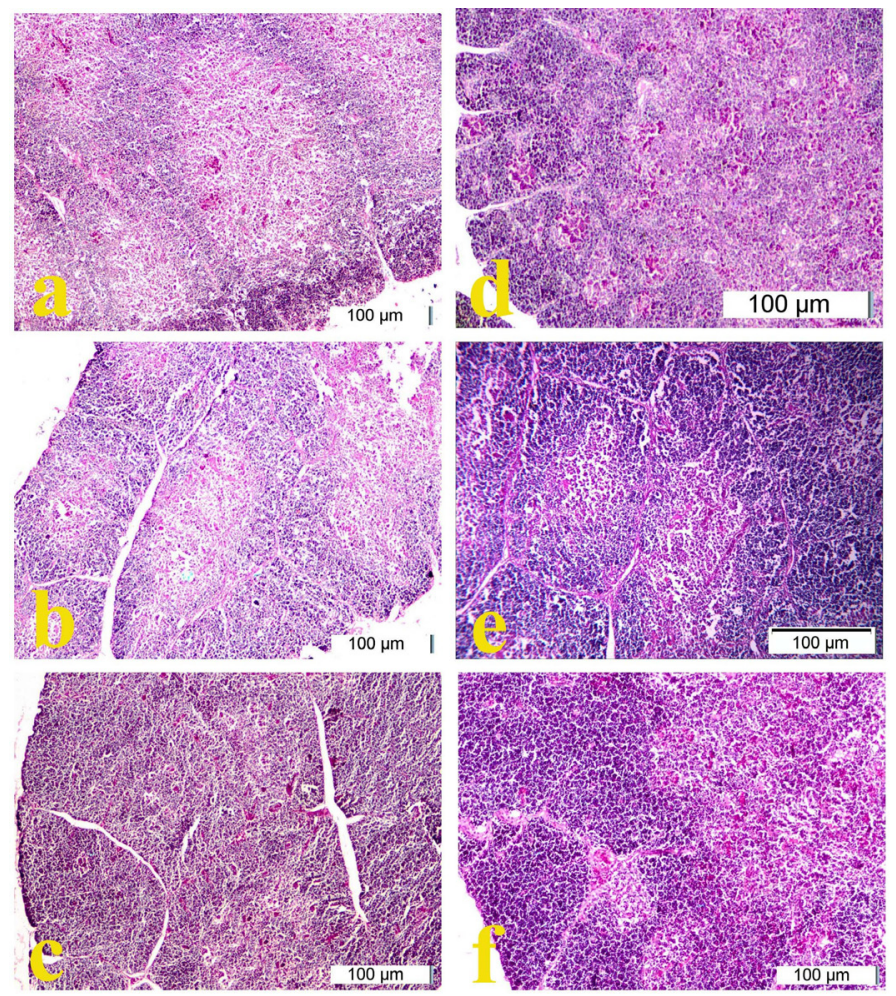

Figure 7 - Representative photomicrographs of Thymus histopathology (10x): (a) Thymus of Arbor Acres ${ }^{\circledR}$ broilers supplemented with $1 \mathrm{~mL}$ nano-selenium/1 L drinking water showing depletion of lymphocytes. (b) Thymus of Arbor Acres ${ }^{\circledR}$ broilers supplemented with $1 \mathrm{~mL}$ selenium/1 L drinking water. (c) Thymus of control Arbor Acres ${ }^{\circledR}$ broilers. (d) Thymus of Ross ${ }^{\circledR} 308$ broilers supplemented with $1 \mathrm{~mL}$ nano-selenium/1L drinking water. (e) Thymus of Ross ${ }^{\circledR} 308$ broilers supplemented with $1 \mathrm{~mL}$ selenium/ $1 \mathrm{~L}$ drinking water. (f) Thymus of control Ross ${ }^{\circledR 3} 08$ broilers. H\&E. Bar $100 \mu \mathrm{m}$.

by $E$. coli O157:H7 $1.6 \times 10^{8}$ challenge on the $10^{\text {th }}$ day of age. The results were in agreement with Jiang et al. (2017) who reported that 0.3 ppm seleniumYeast with $1.5 \%$ soybean or linseed oil was able to enhance carcass quality and oxidative stability even in the presence of stressors. Bakhshalinejad et al. (2018) reported outcomes supporting the current results, they used $0.4 \mathrm{mg}$ SeNPS $/ \mathrm{kg}$ in male Ross broilers and revealed a significant increase in GSH-Px, TAC, and MDA activity and formation.

TBC and TEC were significantly reduced in the intestine and breast muscles in the current study after supplementing Ross ${ }^{\circledR} 308$ and Arbor Acres ${ }^{\circledR}$ broilers with $1 \mathrm{~mL}$ SeNPS $(100 \mathrm{mg} / 1 \mathrm{~L})$. An intergroup variation in TBC and TEC counts with significant increases on the $39^{\text {th }}$ day were attributed to overwhelming microclimatic conditions. The reduction of overall pathogenic bacterial counts might be attributed to the ameliorated immunity conditions. The results agreed with those of Barko et al. (2017) who reported that SeNPS was able to modify intestinal microbiota to compete against pathogenic micro-organisms and restrict their survival. Underwood (2014) recommended a dose of $0.9 \mathrm{mg}$ SeNPS/ $\mathrm{kg}$ to encourage the beneficial growth and proliferation of opportunistic microorganism in broilers' gut to overcome the growth of the pathogenic ones. The recorded antibacterial action of $1 \mathrm{~mL}$ SeNPS $(100 \mathrm{mg} / 1 \mathrm{~L})$ was supported by the in-vitro antibacterial action recorded by Gangadoo et al. (2016) using SeNPS against poultry pathogens including Escherichia coli. Trang et al. (2017) conducted an in-vitro study to evaluate the antibacterial action of SeNPS, in agreement with the current results, they found dose-dependent antibacterial action by using 10 $\mu \mathrm{gSeNPS} / \mathrm{mL}$ against Staphelococcus aureus, Listeria monocytogens, E. coli O157:H7, and Salmonella.

The influence of selenium on productive performance in different broiler breeds are somewhat changeable. In our study, supplementing $1 \mathrm{~mL}$ SeNPS $(100 \mathrm{mg} / 1 \mathrm{~L})$ had an enhancing effect on the productive performance, despite of the individual variation among groups in relation to age. Similar results had been established to other studies as Senthil et al. (2015) and Selim et al. (2015), suggesting that addition of SeNPS both in diets or in drinking water was more effective compared to inorganic selenium to get better performance for broilers. However, our results were in contrary with the results recorded by Rao et al. (2013) and Chen et al. (2013), who recorded in their studies a non-significant difference in growth performance of broilers supplemented with SeNPS.

Our results revealed that $1 \mathrm{~mL}(100 \mathrm{mg} / 1 \mathrm{~L})$ of inorganic selenium and selenium nanoparticles (SeNPs) were not able to enforce enough protection against E. coli pathognomonic lesions and in Arbor Acres ${ }^{\circledR}$ broilers represented as severe perihepatitis and pericarditis that extended to the myocardium resulting in myocarditis. Changes recorded in the liver and heart in the present study are consistent with those of Manimaran et al. (2003). Also, the recorded histopathological lymphoid depletion in bursa, thymus, and spleen was in agreement with the results recorded by Nakamura et al. (1990) who reported that E. coli infection induce damage in the immune systems of the chickens including lymphocyte depletion in both the bursa and the thymus.

Meanwhile, our results revealed a higher degree of protection against $E$. coli lesions in Ross ${ }^{\circledR} 308$ broilers supplemented with $1 \mathrm{~mL}(100 \mathrm{mg} / 1 \mathrm{~L})$ SeNPs, as we recorded mild hepatic lesion, mild pericarditis, and no pathological changes were recorded in the spleen, bursa, and thymus. On the other hand, $1 \mathrm{~mL}(100 \mathrm{mg} / 1$ L) of inorganic selenium in Ross ${ }^{\circledR 3} 308$ broilers gave moderate protection against $E$. coli lesions as mild perihepatitis, mild pericarditis, and mild to moderate 
Ali AA, Soliman ES, Hamad RT, El-Borad OM, Hassan RA, Helal MS
Preventive, Behavioral, Productive, and Tissue Modification using Green Synthesized Selenium Nanoparticles in the Drinking Water of Two Broiler Breeds under Microbial Stress lymphoid depletion of the spleen, bursa, and thymus. These results came inconsistent with Jia et al. (2005) who reported that selenium is an essential trace element that is commonly used as an antioxidant for animals, but there is a very narrow margin between the safe and toxic nutrient levels. Also, Qu et al. (2017) reported that, a new source of selenium is selenium nanoparticles (SeNPs) which exhibit the following properties: antioxidant, antibacterial activities, lower toxicity, better absorption and bioactivity. In addition, SeNPs decreased the oxidative stress in the hens, and improved the avian immune system status by alleviating the immunocompetent cells ability for an antigen challenge.

In the same line, selenium is a necessary trace element, which has antioxidant activity, this has been sustained by both clinical and laboratory experiments (Boostani et al. 2015).

\section{CONCLUSION}

The addition of $1 \mathrm{~mL}$ SeNPS (100 mg/L) rather than inorganic selenium (100 mg/L) per liter of drinking water was able to enhance some aspects of behavioral patterns, biochemical profile, immune and antioxidant functions, and decreased TBC and TEC inline with microbial stress from challenge and surrounding microclimate in Arbor Acres ${ }^{\circledR}$ and Ross $^{\circledR 3} 308$ broilers with enhanced influence in $\operatorname{Ross}^{\circledR 3} 308$ rather than Arbor Acres ${ }^{\circledR}$ broilers.

By the end of this study, results provided too many new question marks to be answered as: can lower doses and concentrations of SeNPS in drinking water produce the same stimulatory influence on the broiler breeds used and other breeds? Can lower doses and concentrations of SeNPS in drinking water minimize the influence of stressors other than biological?

\section{AUTHORS' CONTRIBUTIONS}

ESS designed the experiment, participated in the preparation, executing the experiment, and in writing the manuscript. AAA participated in executing the experiment, and in writing the manuscript. RTH conducted the histopathological examination and participated in writing the manuscript, RAH participated in executing the experiment and in writing the manuscript, OMAB prepared SeNPS, ran the characterization, and participated in writing the manuscript. MSH participated in writing the manuscript.

\section{ACKNOWLEDGMENT}

Sincere thanking should be provided to Prof. MA Sobieh for his directions and guidance during the execution of the experiment and to the community services and environmental development sector, Faculty of Veterinary Medicine, Suez Canal University, Ismailia, Egypt for their help.

\section{COMPETING INTERESTS}

The author declared no conflict of interest.

\section{REFERENCES}

Ahmadi M, Ahmadian A, Seidavi AR. Effect of different levels of nanoselenium on performance, blood parameters, immunity and carcass characteristics of broiler chickens. Poultry Science Journal 2018;6(1):99108 .

APHA - American Public Health Association.Standard methods for the examination of water and wastewater. $22^{\text {th }} \mathrm{ed}$. Washington: American Water Work Association Publication; 2012.

Aparna N, Karunkaran R, Parthiban M. Effect of Selenium Nano Particles on Glutathione Peroxidase mRNA Gene Expression in Broiler Chicken. Indian Journal of Science and Technology 2017;10(32):1-5.

Argyrous G. Statistics for research;with a guide to SPSS. $2^{\text {nd }}$ ed. London: Sage Publications; 2005.

Bakhshalinejad R, Kakhki RAM, Zoidis E. Effects of different dietary sources and levels of selenium supplements on growth performance, antioxidant status and immune parameters in Ross 308 broiler chickens. British Poultry Science 2018;59(1):81-91.

Barko PC, McMichael MA, Swanson KS, Williams DA. The gastrointestinal microbiome: a review. Journal of Veterinary Internal Medicine 2017;32(1):9-25.

Boostani A, Sadeghi AA, Mousavi SN, Chamani M, Kashan N. Effects of organic, inorganic, and nano-Se on growth performance, antioxidant capacity, cellular and humoral immune responses in broiler chickens exposed to oxidative stress. Livestock Science 2015;178:330-336.

Chen $\mathrm{G}, \mathrm{Wu}$ J, Li C. The effect of different selenium levels on production performance and biochemical parameters of broilers. Italian Journal of Animal Science 2013;12(4):486-491.

Darboux R. Histological section realization for the optical microscope Bénin: Faculty of Health Sciences, University of Abomey-Calavi; 1994.

Del Puerto M, Cabrera MC, Saadoun A. A note on fatty acids profile of meat from broiler chickens supplemented with inorganic or organic selenium. International Journal of Food Science 2017;1-8.

El Sabry MI, McMillin KW, Sabliov CM. Nano-technology considerations for poultry and livestock production systems - a review. Annals of Animal Sciences 2018;18(2):319-334.

European Union. List of the authorized additives in feeding stuffs published in application of Article 9t (b) of Council Directive 70/524/ EEC concerning additives in feeding stuffs 2004. Official Journal of European Union 2004;50:1-144.

Ferencik M, Ebringer L. Modulatory effects of selenium and zinc on the immune system. Folia Microbiologica 2003;48 (3):417-426. 


\section{Preventive, Behavioral, Productive, and Tissue Modification using Green Synthesized Selenium Nanoparticles in the Drinking Water of Two Broiler Breeds under Microbial Stress}

Omidi A, Jafari R, Nazifi S, Parker MO. Potential role for selenium in the pathophysiology of crib-biting behavior in horses. Journal of Veterinary Behavior 2018;23:10-14.

Purohit MP, Verma NK, Kar AK, Singh A, Ghosh D, Patnaik S. Inhibition of thioredoxin reductase by targeted selenopolymeric nanocarriers synergizes the therapeutic efficacy of doxorubicin in MCF7 human breast cancer cells. ACS Applied Materials and Interfaces 2017;9(42):3649336512.

Qu W, Yang J, Sun Z, Zhang R, Zho F, Zhang K, et al. Effect of Selenium Nanoparticles on Anti-Oxidative Level, Egg Production andQuality and Blood Parameter of Laying Hens Exposed to Deoxynivalenol. Journal of Animal Research and Nutrition 2017;2(1):1

Rao SVR, Prakash B, Raju MVN, Panda AK, Poonam S, Murthy OK. Effect of supplementing organic selenium on performance, carcass traits, oxidative parameters and immune responses in commercial broiler chickens. Asian Australasian Journal of Animal Sciences 2013;26(2):247-252.

Schwean-Lardner K, Fancher BI, Classen HL. Impact of day length on behavioural output in commercial broilers. Applied Animal Behavior Science 2012;137:43-52.

Selim NA, Radwan NL, Youssef SF, Salah Eldin TA, Abo Elwafa S. Effect of inclusion inorganic, organic or nano selenium forms in broiler diets on:growth performance, carcass and meat characteristics. International Journal of Poultry Science 2015;14 (3):135-143.

Senthil CK, Sugapriya S, Dhayalan V, Ranjithkumar R, Chandarshekar B. Influence of dietary selenium nanowires on growth performance of broiler chicken. International Journal of Biosciences and Nanosciences 2015;2(4): 78-83.

Soliman ES, Hamad RT, Ahmed A. Prophylactic and immune modulatory influences of Nigella sativa Linn. in broilers exposed to biological challenge. Veterinary World 2017;10(12):1447-1455.

Soliman ES, Hassan RA. Evaluation of superphosphate and meta-bisulfide efficiency in litter treatment on productive performance and immunity of broilers exposed to ammonia stress. Advances Animal Veterinary Science 2017; 5(6):253-259.

Soliman ES, Hassan RA. Impact of lighting color and duration on productive performance and Newcastle disease vaccination efficiency in broiler chickens. Veterinary World 2019;12(7):1052-1059.

Soliman ES, Moawed SA, Ziaan AMG. Assessing cleaning and disinfection regime in a slaughterhouse against carcasses contamination. Advances in Animal Veterinary Science 2016;4(9):449-457.

Suchý P, Straková E, Herzig I. Selenium in poultry nutrition: a review. Czech Journal of Animal Science 2014;59(11):495-503

Trang HDN, Vardhanabhuti B, Lin M, Mustapha A. Antibacterial properties of selenium nanoparticles and their toxicity to Caco-2 cells. Food Control 2017;77:17-24

Trukhachev VI, Orobets VA, Skripkin VS, Sevostyanova OI. Comparative analysis efficacy selenium containing vitamin complexes to growing broilers. Research Journal of Pharmaceutical, Biological and Chemical Sciences 2016;7 (3), 2334-2339.

Underwood MA. Intestinal dysbiosis: novel mechanisms by which gut microbes trigger and prevent disease. Preventive Medicine 2014;65:133-137.

Villagra A, Olivas I, Althaus RL, Gomez EA, Lainez M, Torres AG. Behavior of broiler chickens in four different substrates:a choice test. Revista Brasileira De Ciéncia Avicola 2014;16 (1):67-76.

Zamani Moghaddam A, MehraeiHamzekolaei M, Khajali F, Hassanpour H. Role of selenium from different sources in prevention of pulmonary arterial hypertension syndrome in broiler chickens. Biological Trace Element Research 2017;176:1-7. 
